\title{
Article
}

\section{The Epoch of Reionization in Warm Dark Matter scenarios}

\author{
M. Romanello ${ }^{1,2, *}$, N. Menci $^{3}$ and M. Castellano ${ }^{3}$
}

1 Dipartimento di Fisica e Astronomia - Alma Mater Studiorum Università di Bologna, via Piero Gobetti 93/2, I-40129 Bologna, Italy

2 INAF - Osservatorio di Astrofisica e Scienza dello Spazio di Bologna, via Piero Gobetti 93/3, I-40129 Bologna, Italy

3 INAF - Osservatorio Astronomico di Roma, via di Frascati 33, 00040 Monte Porzio Catone, Italy

* Correspondence: massimilia.romanell2@unibo.it

\begin{abstract}
In this paper we investigate how the Reionization process is affected by early galaxy formation in different cosmological scenarios. We use a semi-analytic model with suppressed initial power spectra to obtain the UV Luminosity Function in thermal Warm Dark Matter and sterile neutrino cosmologies. We retrace the ionization history of intergalactic medium with hot stellar emission only, exploiting fixed and mass-dependent photons escape fraction $\left(f_{\text {esc }}\right)$. For each cosmology, we find an upper limit to fixed $f_{e s c}$, which guarantees the completion of the process at $z<6.7$. The analysis is tested with two limit hypothesis on high-z ionized hydrogen volume fraction, comparing our predictions with observational results. We then implement a blast-wave model, which explains the genesis of UV photons escape fraction in the context of feedback and co-evolution between galaxies and Active Galactic Nuclei. Including the AGNs contribution, we find that the neutral hydrogen ionization is almost complete at $z<7$, with a weak dependence on initial gaseous ionized fraction and accretion UV spectral slope.
\end{abstract}

Keywords: Reionization; Warm Dark Matter; sterile neutrinos; escape fraction; AGNs

\section{Introduction}

The Epoch of Reionization (EoR) marked a fundamental phase transition in the history of the Universe, during which the Intergalactic Medium (IGM) became transparent to UV photons. This phase transition is most likely caused by the energetic photons emitted by the first sources of light ever formed, i.e. galaxies and AGNs. A "late Reionization" scenario emerges from the combination of several probes, like the Thomson scattering optical depth of cosmic microwave background (CMB) photons on the free electrons in the IGM [1-3], the absorption due to neutral intergalactic hydrogen and the appearance of Gunn-Peterson thought in the spectra of distant QSOs [4,5], the evolution of the Lyman- $\alpha$ emitters (LAEs) luminosity function [6] and the decreasing of the abundance of the Lyman-Break-Galaxies (LBGs) at $z>6[6,7]$. Although the most recent observations indicate a late-Reionization scenario [2,8,9], with the end of the EoR at $z \approx 6$, the exact contribution from different ionizing sources and the exact timeline and topology of Reionization are still unknown.

From a theoretical point-of-view, cosmic Reionization depends on non-linear, and nonlocal phenomena, in which the physics of galaxy formation couples with the physics of gravity and radiation transport. The first process is determined by both baryonic physics and poorly known feedback effects, but also by the initial power spectrum of density fluctuations: in fact, dark matter produces the potential wells in which baryonic perturbation undergo an accelerated growth. Therefore, the study of Reionization is strongly related to the comprehension of cosmological framework in which cosmic structures form and grow.

The currently most acknowledged cosmological model is the $\Lambda \mathrm{CDM}$ model. It is based on the contribution of the cosmological constant $\Lambda(\approx 69 \%)$ and Cold Dark Matter 
$(\approx 26 \%)$ and provides a coherent large-scale description of the Universe with respect to the available data. However, there are some possible tensions related to observations at galactic and sub-galactic scales, of the order of kpc. In particular, from N-bodies simulations is expected an abundance of galactic sub-halo that does not match with the small number of satellites around the Milky Way $[10,11]$. Furthermore, the simulated halos density profiles are more concentrated with respect to the observed ones [10]. It is possible to try to solve the so-called "missing satellites" problem and "cusp-core" problem within the $\Lambda \mathrm{CDM}$ scenario, with the aid of baryonic effects that act in the faint galaxies [10]. In particular, the supernovae feedback is more efficient in the shallowest potential well, and causes the expulsion of the galactic gaseous content, while the photo-ionization feedback determines the suppression of star formation in small galaxies, because is more difficult for their host halos to collect gas from an ionized environment [10,12].

The $\Lambda$ CDM model postulates the existence of Dark Matter in a "cold" version, i.e. composed by Weakly Interacting Massive Particles (WIMPs) with $m_{X}>0.1 \mathrm{GeV}$ or condensates of light axions, with $m_{X} \approx 10^{-5}-10^{-1} \mathrm{eV}$. The lack of detection of CDM candidates has suggested the possibility to investigate on alternative cosmological scenarios, based on the existence of Warm Dark Matter particles, with mass of the order of $\mathrm{keV}$, which can resolve some of the problems that affect the $\Lambda \mathrm{CDM}$ model at the kpc scales. Indeed, during the structures formation, the motion and the thermal velocity of collisionless DM particles imply the deletion of cosmic perturbations smaller than the "free streaming lenght". While in the $\Lambda \mathrm{CDM}$ model, due to the high mass particles, all the cosmological density perturbations can become gravitationally unstable, in a WDM scenario, depending on the value of $m_{X}$, only perturbations above the kpc scale can collapse, producing shallower density profiles and a smaller number of low-mass halos. This, in the context of the hierarchical growth of the cosmic structures, implies a reduction in the number of faint galaxies and a delay in their formation [13,14]. This fact could have macroscopic consequences on the rest frame galaxy UV Luminosity Function (LF) and several studies are searching for the existence of a possible "turn-over" in its faint end $[12,15]$.

In case of WDM cosmologies, the simplest approach is to consider particles that behave as "thermal relics", resulting from the freeze-out of DM species initially in thermal equilibrium with the early Universe. The suppression of the thermal power spectrum allows to derive constraints to the WDM particles mass, comparing the halo mass function obtained from N-bodies simulations, with the UV luminosity function at high redshift, as performed in Corasaniti et al. (2017), who derives a lower limit $m_{X}>1.5 \mathrm{keV}$ [16]. Viel et al. (2013) found $m_{X}>3.3 \mathrm{keV}$, analyzing the Ly $\alpha$ forests selected from a sample of QSOs [17]. A baryonic-independent estimation of $m_{X}>2.1 \mathrm{keV}$ was found by Menci et al. (2016), using the lensing proprieties of a galaxy cluster to measure the abundances of high-z galaxies and to derive a lower limit to the dark matter halo mass function [13]. A possible alternative is offered by sterile neutrinos (SN) or right-handed neutrinos, which are particles predicted in the context of Standard Model extensions. Since they are produced out-of-equilibrium, from the oscillations of active neutrinos, they are characterized by a non-thermal power spectrum, which depends both on mass and on $\sin (2 \theta)$, where $\theta$ is the mixing angle [18]. Sterile neutrinos can constitute an example of radiatively decay dark matter, potentially explaining the discovery of a $3.5 \mathrm{keV}$ emission line observed towards the center of galaxy and in Perseus, Coma and Ophiuchus Clusters [19].

Some recent works have treated galaxy formation and evolution in a WDM framework. Dayal et al. (2015) found that the suppression of small-scale structures leads to a delayed and more rapid stellar assembly in thermal $1.5 \mathrm{keV}$ WDM [14]. This result was confirmed also for sterile neutrino cosmologies by Menci et al. (2018), which indicate that models with suppressed power spectra are characterized by a delay in the stellar mass growth history, ranging from 500 Myr to 1 Gyr [20]. Reionization in different cosmologies was already discussed in Dayal et al. (2017). Their study pointed out that, in CDM models, 
the bulk of the ionizing photons is produced by systems with $-15<M_{U V}<-10$ and $M_{\text {halo }} \lesssim 10^{9} M_{\odot}$, while in WDM case they registered a shift towards $M_{\text {halo }} \gtrsim 10^{9} M_{\odot}$ and $-17<M_{U V}<-13$, due to the effect of suppression. However, the faster WDM galaxy assembly implies that the reheating of IGM is almost finished at $z \approx 5.5-6$ [21].

In this paper we investigate how Reionization scenarios are affected by early galaxy formation in WDM models. We have used the theoretical model by Menci et al. (2018) [20], where the collapse history of dark matter halos is modelled through the Extended Press-Schechter (EPS) formalism and baryonic processes taking place in each halo are included through physically motivated analytical recipes. We have focused on three thermal WDM scenarios, with masses of $2-3-4 \mathrm{keV}$ and five sterile neutrino models, with a $7 \mathrm{keV}$ mass, but characterized by different lepton asymmetry parameter, $L_{6}$, strongly related to the mixing angle [22].

The paper is organized as follows. Sect. 2 is dedicated to the semi-analytic model and our hypotheses on Reionization history: sect. 2.1 provides a description of the suppression function of the original CDM power spectrum, in sect. 2.2 we outline how to reconstruct the evolution of hydrogen filling fraction with different models of escape fraction, in sect. 2.3 we briefly present the AGN blast-wave model for the calculus of $f_{\text {esc }}$ and its implications. In sect. 3 we describe our results: in sect. 3.1 we show the UV Luminosity function in different WDM scenarios, in sect. 3.2 we present the distribution of $f_{\text {esc }}$ and $\xi_{i o n}^{A G N}$, in sect. 3.3 we evaluate the contribution of faint and bright galaxies with fixed and variable escape fraction models. Sect. 4 is devoted to discussion, which includes the comparison with some authors in literature and with selected observational data (sects. 4.1 and 4.5), the description of Reionization process with galaxies (sect. 4.3) and AGNs (sect. 4.4). Finally, in sect. 5 we have the conclusions.

In this work we adopt the following cosmological parameters: $H_{0}=70 \mathrm{~km} / \mathrm{s} / \mathrm{Mpc}$, $\Omega_{\Lambda}=0.7, \Omega_{m}=0.3$ and $\Omega_{b}=0.045$.

\section{Materials and Methods}

\subsection{Semi-analytic Model}

In our study we use the semi-analytic model developed by Menci et al. (2018), to which we refer for further informations [20]. The model retraces the collapse of dark matter halos through a Monte Carlo procedure on the basis of the merging history given by EPS formalism, at $0<z<10$ [20]. In this framework, the DM structures formation is determined by the power spectrum: the WDM $P(k)$ is computed starting from the CDM cosmology, by the suppression due to the particles free streaming at kpc scale.

An half-mode wavenumber is defined, as the $k_{h m}$ at which the transfer function $T_{W D M}(k)$ is equal to $1 / 2$ [23-25]. For thermal WDM, the power spectra ratio is related to the WDM particle mass $m_{X}$ :

$$
T_{W D M}(k)=\left[\frac{P_{W D M}}{P_{C D M}}\right]^{1 / 2}=\left[1+(\epsilon k)^{2 \mu}\right]^{-5 / \mu},
$$

with $\epsilon$ equal to:

$$
\epsilon=0.049\left[\frac{\Omega_{X}}{0.25}\right]^{0.11}\left[\frac{m_{X}}{k e V}\right]^{-1.11}\left[\frac{h}{0.7}\right]^{1.22} \frac{h^{-1}}{M p c},
$$

where $\Omega_{X}$ is the density parameter of DM and $\mu=1.12$ [13][20].

From eq. 1 we can also define an half-mode mass $M_{h m}$ :

$$
M_{h m}=\frac{4 \pi}{3} \rho_{m}\left[\pi \epsilon\left(2^{\mu / 5}-1\right)^{-1 / 2 \mu}\right]^{3}
$$

Conversely, for sterile neutrino based cosmological scenarios, we refer to $M_{h m}$ from Lovell et al. (2020), obtained comparing CDM and WDM simulations performed within 
the same cosmic volume and in which the parameterization of the WDM halo mass function is given by $R_{\text {fit }}$ [26]:

$$
R_{f i t}=\frac{n_{W D M}}{n_{C D M}}=\left(1+\left(\alpha \frac{M_{h m}}{M_{h a l o}}\right)^{\beta}\right)^{\gamma},
$$

where $n_{C D M}$ and $n_{W D M}$ are the differential halo mass functions and $M_{\text {halo }}$ is the halomass. The numerical value of $\alpha, \beta$ and $\gamma$ coefficients changes if we consider central $(\alpha=2.3, \beta=0.8, \gamma=-1.0)$ or satellite halos $(\alpha=4.2, \beta=2.5, \gamma=-0.2)$ [26].

We perform our analysis with five different sterile neutrino models, with a mass of 7.0 $\mathrm{keV}$, labelled according to the lepton asymmetry number $\left(L_{6}\right)$, which is indicated in the last part of the name. For example, $L_{6}=120$ is named LA120, $L_{6}=8$ is named LA 8 and so on. Among them, the models LA9, LA10 and LA11 are based on decaying-particles that are compatible with the X-ray $3.55 \mathrm{keV}$ emission line observed in galaxy clusters [26]. In table 1 we also present $M_{h m}$ of three thermal WDM scenarios, with $m_{X}=2-3-4$ $\mathrm{keV}$, computed using eq. 3 .

Table 1. Half-mode mass $\left(M_{h m}\right)$ for each Dark Matter model analysed in this work.

\begin{tabular}{cc}
\hline DM model & $M_{h m}\left(M_{\odot}\right)$ \\
\hline LA8 & $1.3 \times 10^{8}$ \\
LA9 & $2.6 \times 10^{8}$ \\
LA10 & $5.3 \times 10^{8}$ \\
LA11 & $9.2 \times 10^{8}$ \\
LA120 & $3.1 \times 10^{9}$ \\
WDM 2 & $1.6 \times 10^{9}$ \\
WDM 3 & $4.1 \times 10^{8}$ \\
WDM 4 & $1.6 \times 10^{8}$ \\
\hline
\end{tabular}

\subsection{Modelling Reionization}

In the last few years, the completion of deep surveys with efficient IR telescopes has enabled the study of number density and emission properties of distant galaxy populations. In particular, gravitational lensing turned out as an effective tool to achieve the detection of high-redshift galaxies, leading to robust constraints on the observable UV luminosity function at both the bright and the faint end. The UV LF is particularly important because it's strongly related to star formation rate $[27,28]$ and so on the existence of an hot stellar population that acts as a ionizing source responsible of Reionization. The progressive steepening with high redshift in the UV LF faint-end slope confirms the relevant role of faint galaxies in the reheating of IGM [29,30].

The semi-analytic model associates a galactic luminosity to each halo. Thus, from the suppressed halo mass function we can calculate the galaxy luminosity function in any cosmological scenario. In our analysis, we integrate the rest-frame UV $(\sim 1400 \AA)$ dust-corrected LF between the limits $M_{U V}^{l i m}=[-25,-12]$, to obtain the corresponding luminosity density:

$$
\rho_{U V}=\int^{M_{U V}^{l i m}} \mathrm{~d} M_{U V} \frac{\mathrm{d} N}{\mathrm{~d} M_{U V}} R_{f i t} L_{U V},
$$

which is dominated by the contribution of systems with $M_{U V} \geq-20$ (see section 3.3). The number density of UV photons that actively participate to hydrogen ionization process is obtained by multiplying for two quantities [31]:

$$
\dot{N}_{\text {ion }}=f_{\text {esc }} \xi_{\text {ion }} \rho_{U V}
$$

The ionizing photon production efficiency $\left(\xi_{i o n}\right)$ is expressed in $\mathrm{erg} / \mathrm{Hz}$ units and describes how efficiently it's possible to get UV ionizing photons from an UV continuum 
radiation field. This quantity depends on different astrophysical parameters which potentially could alter the temperature of emitting stellar population, as metallicity, initial mass function (IMF), age and binarity [31]. During our analysis, when galaxies are the only source of photons, we use $\xi_{\text {ion }}$ without any specific superscript and we explore some fixed value around $\log \xi_{\text {ion }} \approx 25$ (see sec. 4.3.1).

Finally, the escape fraction $f_{\text {esc }}$ converts the intrinsic ionizing emissivity $\dot{N}_{\text {ion, intrinsic }}=$ $\xi_{i o n} \rho_{U V}$ into an effective one. It is defined as the fraction of ionizing photons which can escape from the source galaxy instead of being reabsorbed inside of it and so actively participates to the ionization of IGM. Due to the difficulty in detection of Ly-C photons $(\lambda<912 \AA)$ for $z>4$ and to the scarce knowledge of the interstellar medium (ISM) geometry of high-z galaxies, this parameter is not actually well constrained and summarizes most of our uncertainties about the EoR.

In our study, we model the Reionization history with different values of $f_{\text {esc }}$. Fixed escape fraction is useful to broadly characterize the Reionization history, although a universal value for $f_{\text {esc }}$ is highly unrealistic. Galaxies represent in fact a very complex sample, with large differences in morphology, mass, luminosity, colour, SFR, gas content, age and metallicity.

Nevertheless, the investigation of the degenerate quantities $f_{\text {esc }} \xi_{\text {ion }}$, which drive the Reionization process, can yield to interesting upper limits to the escape fraction.

We have repeated our investigation introducing a dependence between escape fraction and halo-mass. We use the $f_{\text {esc }}\left(M_{\text {halo }}\right)$ model computed in Yajima et al. (2011), which combines the results of hydrodynamic simulations with radiative transport calculations of stellar radiation, also evaluating the effects of dust extinction, for mass halos in the range $10^{9}-10^{11} M_{\odot}$ [32]. In particular, $f_{\text {esc }}$ shows a noticeable decrease as $M_{\text {halo }}$ increases. From a physical point of view this fact is justified as a consequence of the lower gaseous column density in less massive halos and it is valid in every redshift bin between 3 and 6; however $z$ does not alter significantly the $f_{\text {esc }}$ value [32].

Table 2. Table from Yajima et al. (2011) [32] with dependence between escape fraction and halo-mass.

\begin{tabular}{cc}
\hline $\log M / M_{\odot}$ & $f_{\text {esc }}$ \\
\hline 9 & 0.325 \\
9.5 & 0.212 \\
10 & 0.115 \\
10.5 & 0.132 \\
11 & 0.031 \\
\hline
\end{tabular}

Once obtained $\dot{N}_{i o n}$, the equation that describes the overall balance between ionization and recombination, which regulate the evolution of the hydrogen filling fraction $Q_{H I I}$ is:

$$
\dot{Q}_{H I I}=\frac{\dot{N}_{i o n}}{\bar{n}_{H}}-\frac{Q_{H I I}}{t_{r e c}}
$$

where the comoving hydrogen mean density is computed as $\bar{n}_{H} \approx 2 \times 10^{-7}\left(\Omega_{b} h^{2} / 0.022\right)$ $\mathrm{cm}^{-3}$ and the recombination time-scale is $t_{\text {rec }} \approx 3.2 \mathrm{Gyr}[(1+z) / 7]^{-3} C_{\text {HII }}^{-1}$ [33]. We consider case $\mathrm{B}$ of recombination.

We treat the evolution of the clumping factor $C_{H I I}$ with redshift, due to the effect of UVB generated by Reionization, according to [34,35]:

$$
C_{H I I}=1+43 z^{-1.71} \text {. }
$$


After the reconstruction of the Reionization history, we use the redshift evolution of the filling fraction to compute the integral:

$$
\tau_{e s}(z)=c \sigma_{T} \bar{n}_{H} \int_{0}^{z} Q_{H I I}\left(z^{\prime}\right)\left(1+z^{\prime}\right)^{2}\left(1+\frac{\eta Y}{4 X}\right) H^{-1}\left(z^{\prime}\right) \mathrm{d} z^{\prime},
$$

in which helium is singly-ionized $(\eta=1)$ at $z>4$ and doubly-ionized $(\eta=2)$ at $z<4$ [36]. Then, the electron scattering optical depth has been compared with observational constraint on $\tau_{e s}$ obtained, from CMB anisotropy, by Planck and WMAP.

\subsection{The role of AGNs in EoR: the blast-wave model}

Recent observations have demonstrated that galaxy evolution is affected by feedback from Active Galactic Nuclei, which interact with their host in a radiative and a mechanical way. In particular, evidence of Warm Absorbers and relativistic Ultra Fast Outflows were found in several X-ray spectra of luminous AGNs [37]. The relativistic outflows determine the compression of the intragalactic gas in a blast wave and the formation of a shock that wipes out the interstellar medium. The model presented by Menci et al. (2019), to which we remand for further informations, provides a compact two-dimensional description for the expansion of AGN-driven shocks in galactic disks [38]. It also allows to explore the dependence of the outows from AGN luminosity and from galactic gas mass fraction. In synthesis, the blast wave sweeps away the internal regions of galactic systems, causing a decrease in the gaseous column density. In parallel, it promotes the accumulation of ISM on a thin layer close to the shock. The result is that after the outburst, the same line of sight will intercept only a tiny fraction of the initial gas content [39]. Finally, the general warming of the baryons determines also their ionization. This fact imply, depending on the efficiency of the energy transmission and cooling mechanism, an additional channel for the escape of photons responsible for Reionization.

Starting from AGN bolometric luminosity $L_{b o l}^{A G N}$, from mass and from the cold gas content of each galaxy, we implement the blast-wave model in our semi-analytic framework obtaining, for each active nucleus, the opening angle $\beta$ subtended by the free gas cone produced during shock expansion. The escape fraction is then simply $f_{e s c}=\frac{\beta}{90}$.

With the blast-wave model it's possible to explain the genesis of $f_{\text {esc }}$ and to associate its value both to galaxy and to AGN. The number density of UV photons per unit of time is then composed by the galactic and by the AGN luminosity density:

$$
\dot{N}_{i o n}=\int^{M_{U V}^{l i m}} \mathrm{~d} M_{U V} \frac{\mathrm{d} N}{\mathrm{~d} M_{U V}}\left(L_{U V}^{A G N} \xi_{i o n}^{A G N}+L_{U V}^{g a l} \xi_{i o n}^{g a l} R_{f i t}\right) f_{e s c} .
$$

Computing the integral 10, we sum the contribution of all galactic system between $-12<M_{U V}<-25$. Since only a fraction of galaxies host an AGN in the centre, and since, in our model, a non-zero $f_{\text {esc }}$ can be produced only if $L_{b o l}^{A G N}$ is between $10^{43}$ and $10^{46.6} \mathrm{erg} / \mathrm{s}$, we use the same luminosity function for both sources, because the contribution of quiet galaxies is canceled by $f_{e s c}=0$.

Galaxies and AGNs have a different $\xi_{\text {ion }}$ value. As discussed in sect. 4.3.1, we use the $\xi_{i o n}^{g a l}$ expected from a low metallicity single stars population. Indeed, for the calculation of $\xi_{i o n}^{A G N}$, we construct the AGNs spectra combining formulae by Grissom et al. (2014) and Matsuoka et al. (2018) [40,41].

In particular, every AGN has a proper black hole mass $\left(M_{B H}\right)$, from which it's possible to derive the black-body temperature associated with the accretion [40]:

$$
T_{B B}=1.37 \times 10^{7}\left(\frac{M_{B H}}{M_{\odot}}\right)^{-\frac{1}{4}} K .
$$


The AGN spectrum is then represented using the shape of a power-law with an exponential cut-off:

$$
L_{v}=L_{1400}\left(\frac{v}{v_{1400}}\right)^{\alpha_{U V}} \exp \left(\frac{-h\left(v-v_{1400}\right)}{k_{b} T_{B B}}\right) .
$$

Here, the black-body temperature allows us to take account of the differences in the single AGN spectral energy distribution.

As found by Lusso et al. (2015), analyzing the spectra of a sample of 53 luminous quasars at $z \approx 2.4$ [42], the UV power-law slope has a value of $\alpha_{U V}=-1.70 \pm 0.61$ at $\lambda \leq 912 \AA$. The UV photons production efficiency is then [41]:

$$
\xi_{\text {ion }}^{A G N}=\frac{1}{L_{1400}} \int_{v_{L L}}^{v_{4 L L}} \frac{L_{v}}{h v} \mathrm{~d} v
$$

and it is computed between $v_{L L}$ and $v_{4 L L}$, which are respectively the Lyman limit for ionization of neutral hydrogen and the HeII ionization frequency [41].

\section{Properties of ionizing sources}

\subsection{UV Luminosity Function}

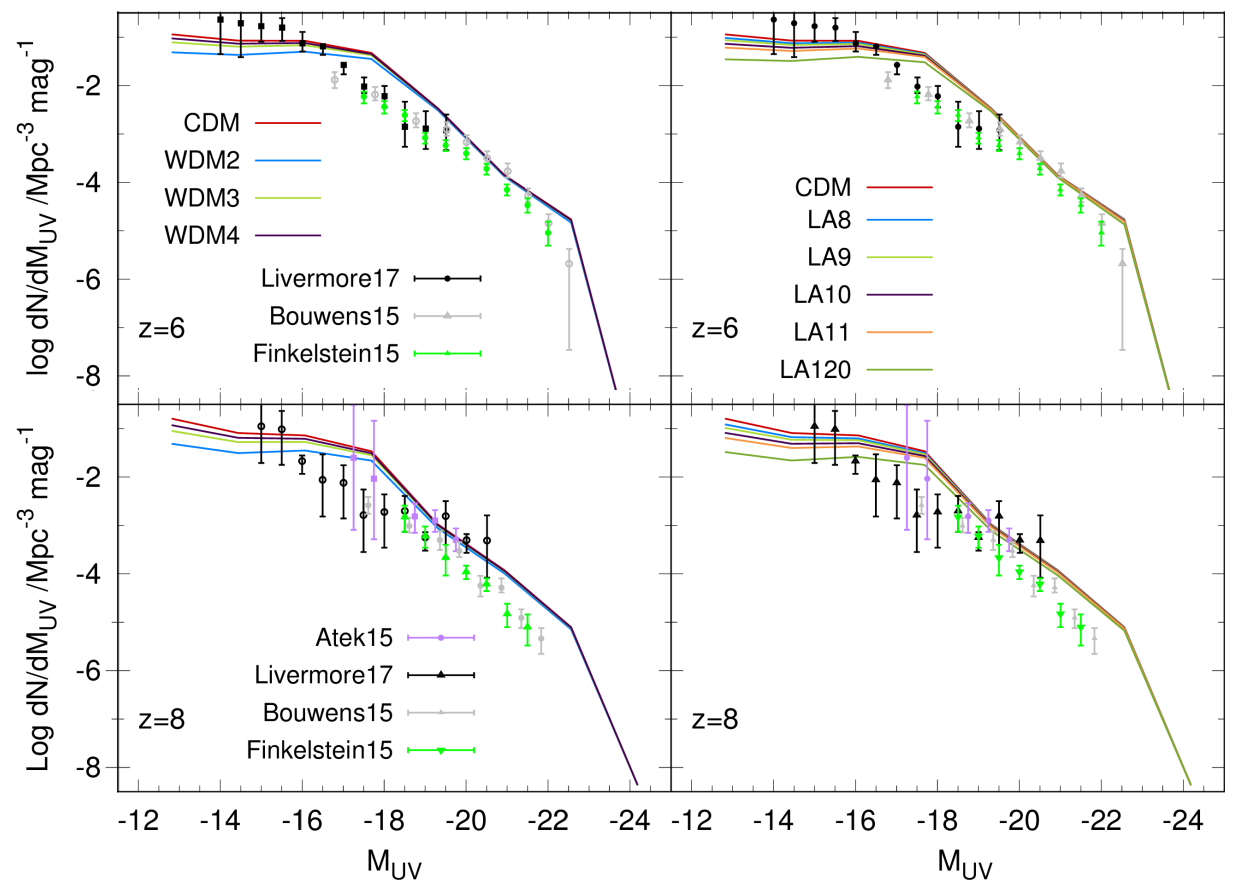

Figure 1. UV LF in different cosmologies, computed using the semi-analytic model, with Calzetti dust extinction law. (Left): comparison between CDM and 2-3-4 keV thermal WDM scenarios. (Right): comparison with sterile neutrino scenarios. Observational constraints are taken from Atek et al. (2015) [29] (purple error bars), Livermore et al. (2017) [30] (black error bars), Bouwens et al. (2015) [27] (grey error bars) and Finkelstein et al. (2015) [28] (green error bars).

In figure 1 we show the UV rest frame luminosity function at $1400 \AA$ in different cosmological scenarios. It is computed with the semi-analytic model in the magnitude interval $-25<M_{U V}<-12$.

From a physical point of view, the free-streaming of dark matter particles suppresses, on average, the formation of the smallest halos, whereas the most massive perturbations are still able to collapse. For this reason, comparing CDM with sterile neutrino and thermal WDM cosmologies, we find a reduction in the comoving galaxy density at higher absolute UV magnitude, with the increasing of the half-mode mass, $M_{h m}$. Although this effect is more evident for thermal WDM with $m_{X}=2 \mathrm{keV}$ (WDM2) and for sterile 
neutrino LA120, it is still insufficient to discriminate between models, with the current observational constraints on UV LF.

WDM galaxy formation is also characterized by an initial delay in the halos collapse and consequently in the star formation process, which manifests itself at lower redshift [43]. Star formation is however more rapid with respect to CDM scenario [14]; this fact progressively reduces the gap between cosmologies with the age of universe, as we can see from figure 1 , looking at $z=8$ and $z=6$ LFs. The result is that differences in the UV LFs become negligible in the local universe.

We have compared our LF with some recent high-z observations performed with Hubble Space Telescope. First, the paper by Atek et al. (2015) is based on the lensing properties of three galaxy cluster: A2744, MACS 0416 and MACS 0717, and on a sample of 25 galaxies at $z \approx 8[29]$.

Finkelstein et al. (2015) indeed analyze the rest frame UV LF between $4<z<8$, with a set of approximately 7500 candidates, obtained by exploiting the galaxy clusters Abell 2744 and MACS J0416.1 - 2403 [28].

We report also the paper by Bouwens et al. (2015), based on observations of 10000 galaxies at $z \geq 4$ and by Livermore et al. (2017), in which a catalogue of 167 objects is used $[27,30]$.

These studies found a steepening in the faint-end of the UV LF, which enforce the role of faint galaxies in the Reionization process. However, the current measurements and uncertainties do not allow us to exclude any cosmological scenario, neither the most suppressed ones. Previous studies did not find evidence of a turn-over in the luminosity function, which could be explained in WDM cosmologies [12,15].

\section{2. $\xi_{\text {ion }}$ and $f_{\text {esc }}$ from $A G N s$}

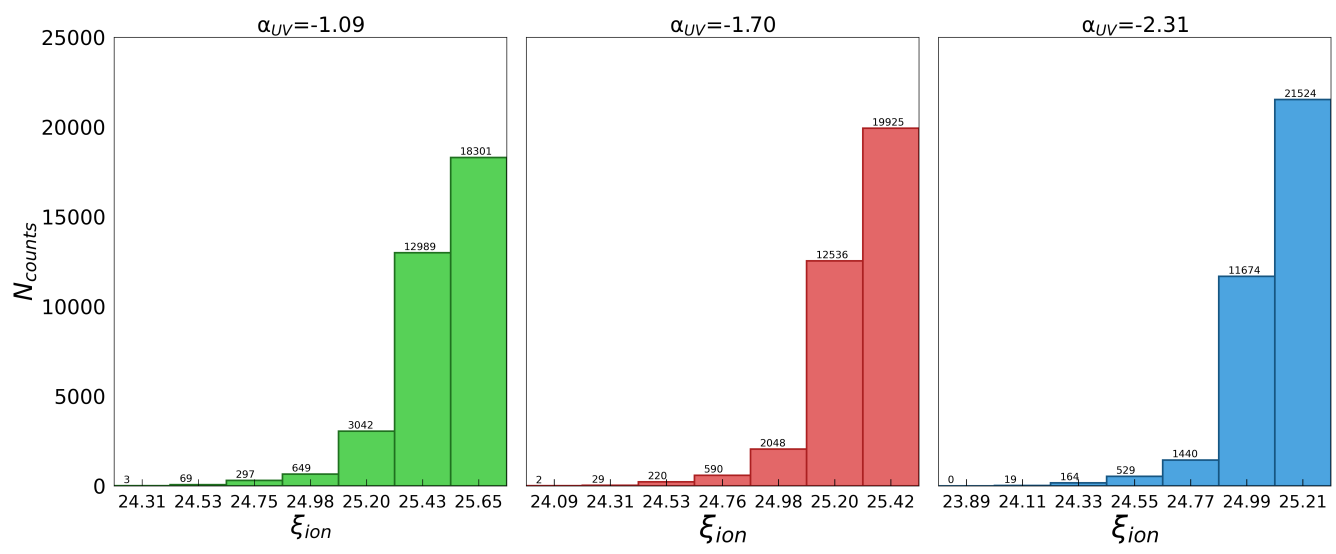

Figure 2. The multiple panels show the distribution of $\xi_{i o n}^{A G N}$, obtained from eq. 13, with different power-law slope. The quantity is evaluated at the upper, the lower and the middle value of the interval $\alpha_{U V}=-1.70 \pm 0.61$, derived by Lusso et. al (2015) [42].

In fig. 2 we show the $\xi_{i o n}^{A G N}$ distribution, computed with eq. 13. A steepening in the power-law slope $\alpha_{U V}$ is associated with a softer UV spectrum and reduces the ionizing photons production. Thus, the peak of the distribution is shifted towards lower $\xi_{i o n}^{A G N}$ values. In fig. 3 we find that the escape fraction distribution exhibits a bimodality, with a broad correlation between the AGN bolometric luminosity and the escape fraction. While faint AGNs are spread in a large $f_{e s c}$ interval, in which the bulk of the systems has $f_{\text {esc }} \approx 0$, the bright-end of our AGN luminosity distribution has $f_{\text {esc }} \geq 0.7$.

This result agrees with the Lyman Continuum observations by Grazian et al. (2018), which studied the emission of 16 AGNs between $3.6<z<4.2$, with an absolute UV magnitude within $-25.1 \lesssim M_{U V} \lesssim-23.3$. They found an escape fraction between $44 \%$ and $100 \%$, with a mean value of $74 \%$ [44]. 

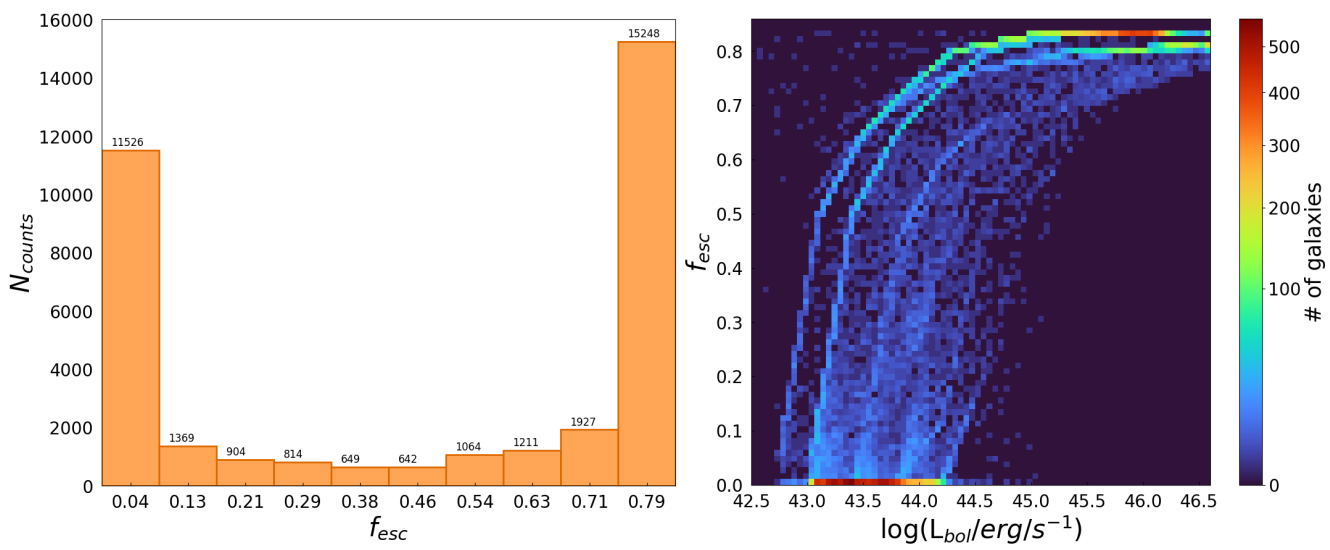

Figure 3. (a) Left: distribution of the $f_{\text {esc }}$ computed with the blast-wave model. (b) Right: 2D histogram with the correlation between the AGN bolometric luminosity and $f_{\text {esc }}$.

3.3. The role of bright and faint galaxies in the Epoch of Reionization

3.3.1. Fixed escape fraction
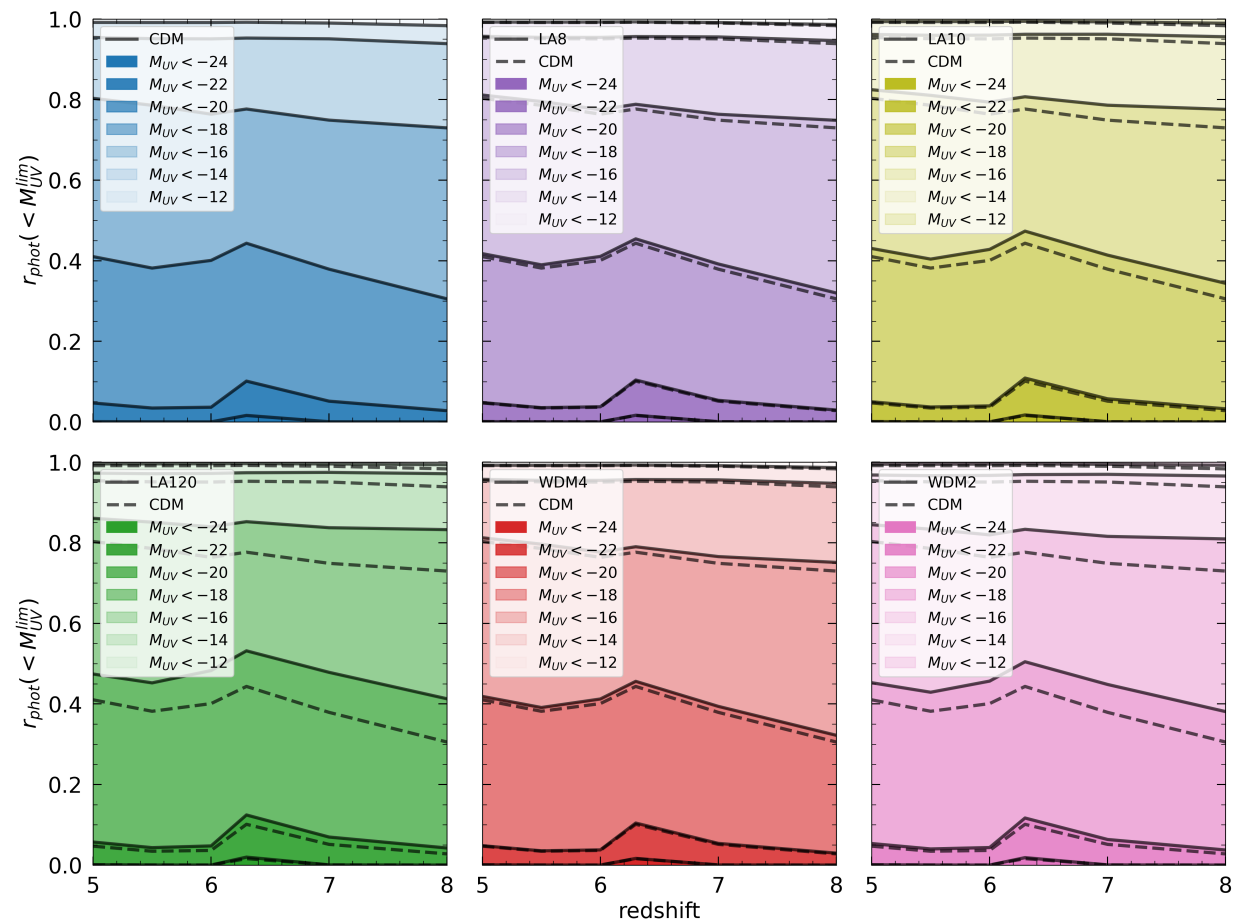

Figure 4. The multiple panels show the integrated photons ratio $r_{\text {phot }}\left(<M_{\text {lim }}^{U V}\right)$, where $\dot{N}_{\text {ion,tot }}$ is computed integrating eq. 5 between $M_{U V}^{\text {sup }}=-12$ and $M_{U V}^{\text {inf }}=-25$. We compare with CDM two thermal WDM cosmologies (WDM3 is an intermediate case bewteen WDM4 and WDM2), and three sterile neutrinos cosmologies (here LA10 is the only representative scenario for radiatively decay Dark Matter, compatible with the $3.5 \mathrm{keV}$ emission line observed in galaxy clusters).

In fig. 4 we plot the integrated ionizing photons ratio:

$$
r_{\text {phot }}\left(<M_{\text {lim }}^{U V}\right)=\frac{\dot{N}_{\text {ion }}\left(M_{U V}<M_{U V}^{l i m}\right)}{\dot{N}_{\text {ion,tot }}}
$$

in which we compute $\dot{N}_{i o n, t o t}$, using eq. 5 between $M_{U V}^{\text {sup }}=-12$ and $M_{U V}^{\text {inf }}=-25$, while the numerator is obtained by varying the upper limit of the integral from -24 to -12 , including so the photons from progressively dimmer sources, until the unity is reached. 
From fig. 4 we can identify two important features, through which we can understand the role and the different contribution of faint and bright galaxies during EoR.

The first is the increasing of the relative contribution of the brighter systems (respectively with $M_{U V}^{\text {sup }}<-24, M_{U V}^{\text {sup }}<-22$ and $M_{U V}^{\text {sup }}<-20$ ) with the age of universe. In the $\Lambda$ CDM model, $r_{\text {phot }}(<-22)$ passes from $2.8 \%$ at $z=8$, to $10 \%$ at $z=6.3$. In parallel, for $M_{u V}^{\text {sup }}=-20$ we have a raise from $31 \%$ at $z=8$ to $44 \%$ at $z=6.3$. We can interpret this trend in the light of the hierarchical growth of cosmic structures: merging phenomena between galaxies give origin to more massive and brighter structures, increasing their overall contribution. However, the role of faint galaxies in the Reionization process is still predominant.

The second issue to be highlighted derive from a comparison between different cosmological scenarios, which reveals that WDM models present a relative $\dot{N}_{i o n}$ higher than the $\mathrm{CDM}$ ones. Again, the reason resides in the effect of free-streaming, which determines a suppression in the number density of the faint-galaxies and so a decreasing in their relative contribution for each $M_{U V}^{\text {sup }}$. As found by Dayal et al. (2017), the progressive suppression of low-mass halos in WDM models produces a shift in the Reionization population to larger halo and galaxy masses [21].

The difference between cosmologies is summarized in the half-mode mass and is not negligible: if we compare CDM with LA8 and WDM4, at $z=8$ it values $\approx 1-2 \%$, respectively for $M_{U V}^{\text {sup }}=-20$ and -18 , but it increases to $8-10 \%$ for WDM2 and LA120. Finally, we noted that the continue (WDM) and the dashed (CDM) lines in fig. 4 approach each other with time; for example, at $z=5$ the differences between CDM and WDM2-LA120 reduce respectively to $4-6 \%$. Again, we can interpret this result by looking at the evolution of the UV LFs with $z$.

We should note however, that the current analysis is independent from the escape fraction value: in fact $f_{\text {esc }}$ appears only as a multiplicative constant, so it simplifies in the ratio between $\dot{N}_{i o n}$. Conversely, if we consider other escape fraction dependencies, we could expect a more various behaviour.

\subsubsection{Variable escape fraction}
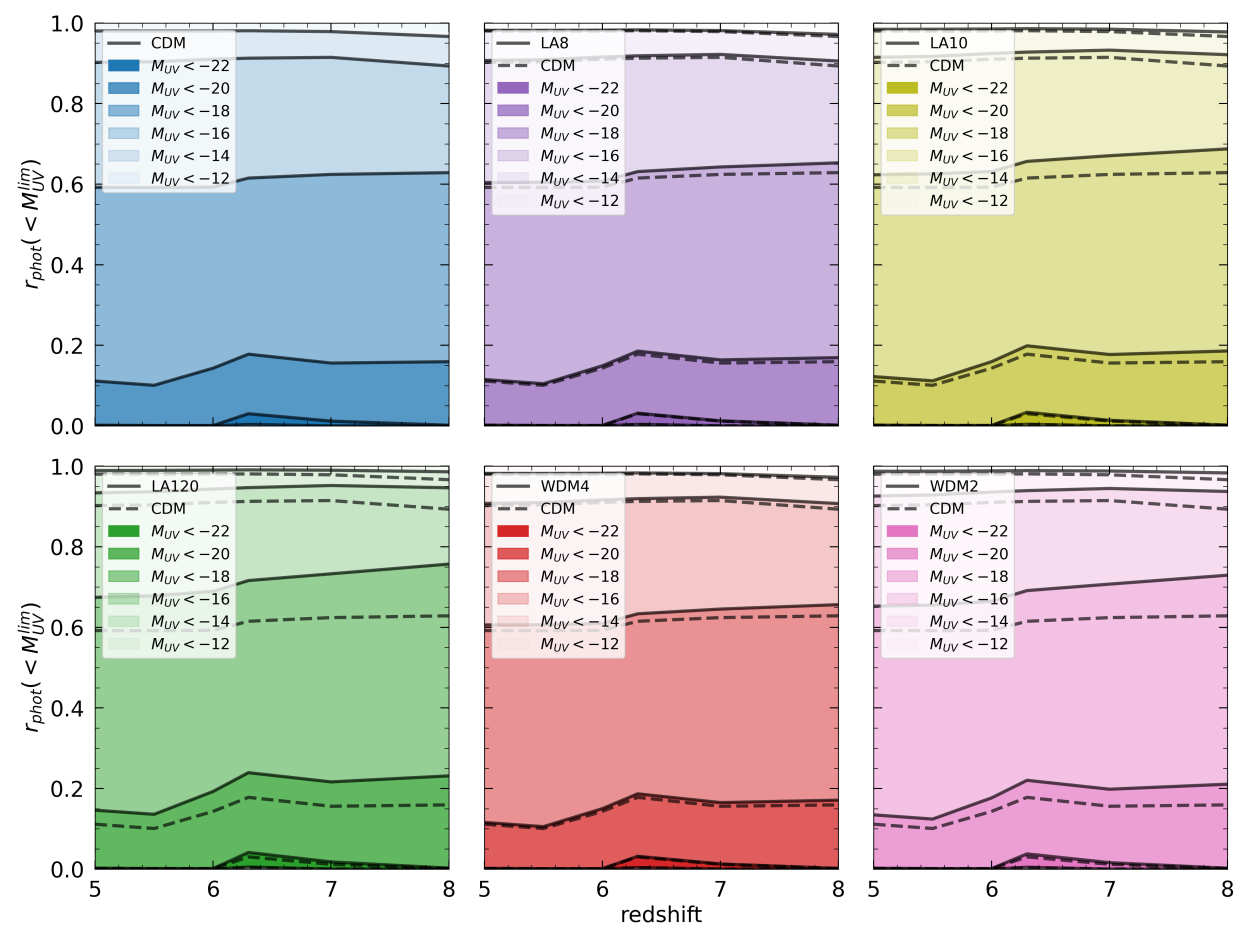

Figure 5. Same as fig. 4, but with the integrated photons ratio $r_{\text {phot }}\left(<M_{\text {lim }}^{U V}\right)$ computed using $f_{\text {esc }}\left(M_{\text {halo }}\right)$ model by Yajima et al. (2011). [32] 
We now show the integrated photons ratio computed assuming the dependency of escape fraction with the halo-mass, assuming the model by Yajima et al. 2011 [32]. In this case $f_{\text {esc }}$ does not act simply as a multiplicative constant; indeed it enters directly into the integral 5, since the most massive halos host in general the most massive galaxies, which are also more luminous.

In fig. 5 we therefore observe a significant reduction in the contribution of bright galaxies with $M_{U V}^{\text {sup }}<-20$, which for CDM cosmology is constantly below $18 \%$. Furthermore, the effect of galaxy-galaxy merging is neutralized by the reduction of $f_{\text {esc }}$ in high-mass systems. We also observe that the cumulative fraction of $\dot{N}_{i o n}$ needs the addition of galaxies with $-14<M_{U V}<-12$ to saturate: these weak sources provide to Reionization process a photons fraction that goes from $2 \%$ for $z=5$, to $4 \%$ for $z=8$.

\subsubsection{Blast-wave model escape fraction}
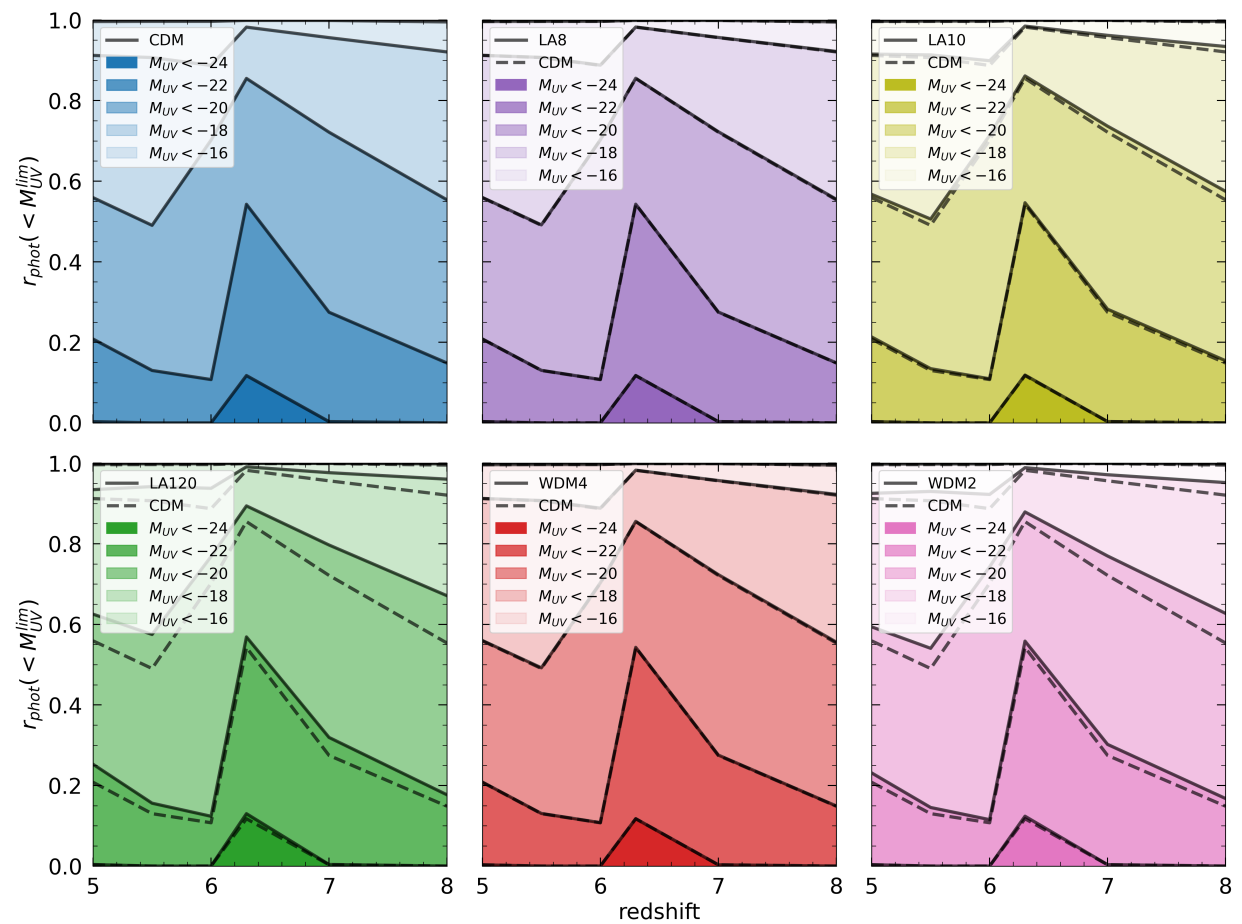

Figure 6. Same as fig. 4, but with the integrated photons ratio $r_{\text {phot }}\left(<M_{\text {lim }}^{U V}\right)$ computed using $f_{\text {esc }}$ AGN blast-wave model.

In fig. 6 we plot the $r_{\text {phot }}\left(<M_{U V}^{\text {lim }}\right)$ ratio, including exclusively the photons produced by galaxies, with no contribution from AGNs. This approach offers the possibility to explore the consequences of the blast-wave model on the relative contribution of bright and faint sources.

In sect. 3.2 we plot the bimodal distribution of escape fraction, finding that a huge number of sources, the most luminous ones, have $f_{\text {esc }} \approx 0.7$. This fact has three significant consequences. First, it enhances the importance of merging between galaxies, explaining the more pronounced steepening in $r_{\text {phot }}$ between $6.3<z<8$. Second, we note that bright galaxies contribute more to Reionization process: even galaxies with $M_{U V}<-24$ produce approximately the $12 \%$ of total ionizing photons at $z \approx 6.3$, while the contribution of sources with $M_{U V}<-22$ and $M_{U V}<-20$ reaches respectively the $55 \%$ and $85 \%$. In the blast-wave model, faint galaxies are relegated to a perturbative role during EoR and do not govern the evolution of the filling fraction. Finally, due to the fact that the bulk of ionizing photons are produced into the most luminous systems, the differences between cosmologies are minimized. As we note also in sect. 4.3.2, the 
blast-wave model introduces a degeneracy between CDM and less suppressed WDM scenarios.

\section{Results and Discussion}

\subsection{Observational Constraints to $Q_{H I I}$}

It is known that the intensity of $\operatorname{Ly} \alpha$ emission line rapidly decreases over $z \approx 6$ [6]. This fact is typically explained with the appearance of neutral hydrogen pockets in the IGM, as shown in some relevant past works available in literature. Here we present a brief summary of some recent observational constraints on $Q_{H I I}$.

Mason et al. (2019) found a lower limit of $Q_{H I}=1-Q_{H I I}>0.76$, starting from the study of Ly $\alpha$ emission in a sample of 29 LBGs with photometric redshift within $z=7.9 \pm 0.6$, plus 8 LBGs already observed at $z \approx 8$ [9].

The previous paper by Mason et al. (2018) is based indeed on a cosmological simulation which describes the IGM large scale structures during the EoR. The analysis of damping wings absorption along different line of sight allows to link its simulated properties to observations on a set of 68 LBGs (collected by Pentericci et al. (2014) [7]), with $-22.75<M_{U V}<-17.8$. The resulting HII volume fraction is $0.59_{-0.15}^{+0.11}$ [45].

The same model was than reimplemented in Hoag et al. (2019), with a different LBGs sample, leading to a neutral hydrogen fraction of $Q_{H I}=0.88_{-0.10}^{+0.05}$, in $z=7.6 \pm 0.6$, which clearly point to a late-Reionization scenario [8].

In fig. 8, 9 and 12, we present under the name "Ly $\alpha$ LF" a series of publications based on the LAEs luminosity function: Konno et al. (2014), estimated $0.3<Q_{H I}<0.8$ at $z=7.3$, Konno et al. (2017) measured $Q_{H I}=0.3 \pm 0.2$ at $z=6.6$ on a group of 1266 LAEs, and Zheng et al. (2017) found $0.4<Q_{H I}<0.6$ at $z=6.9$ [46-48].

Mesinger et al. (2015) used a color selected sample of 56 galaxies from Very Large Telescope spectroscopy; connecting large-scale semi-numeric simulations of Reionization with moderate-scale hydrodynamic simulations of the ionized IGM and performing a study on more than 5000 line of sight, they set an upper limit of $Q_{H I I}(z \approx 7) \leq 0.6$, within a $68 \%$ confidence level [49].

Using the same sample of Konno et al. (2018), Ouchi et al. (2017) obtained the constraint of $Q_{H I}=0.15 \pm 0.15$ at $z=6.6$, by the comparison between clustering measurements of LAEs and two independent theoretical models [50].

Finally, we report the results by Schenker et al. (2014), which used a set of 451 LBGs to study the correlation between the UV continuum slope of a galaxy and its Ly $\alpha$ emission. Combining a cosmological simulations with $\operatorname{Ly} \alpha$ visibility data, they constrained a neutral fraction of $Q_{H I}=0.39_{-0.09}^{+0.08}$, which suggests important evidence that cosmic Reionization ended at $z \approx 6.5$ [51].

Following the evolution of $Q_{H I I}$ with $z$, we compute the electron scattering optical depth with eq. 9. The results are compared with the CMB measurements performed by Planck and WMAP. In table 3 we summarize the observational constraints on $\tau_{e s}$ and the corresponding instantaneous redshift of Reionization [1-3].

Table 3. Electron scattering optical depth and instantaneous redshift of Reionization, measured by WMAP and Planck.

\begin{tabular}{ccc}
\hline Name & $\tau_{\text {es }}$ & $z_{\text {reion }}$ \\
\hline WMAP 9 & $0.088 \pm 0.013$ & $10.5 \pm 1.1$ \\
Planck 2015 & $0.066 \pm 0.016$ & $8.8_{-1.4}^{+1.7}$ \\
Planck 2018 & $0.054 \pm 0.007$ & $7.7 \pm 0.7$ \\
\hline
\end{tabular}

\subsection{Initial condition for late Reionization scenario}

The evolution of the filling fraction with cosmic time depends also on the initial condition for eq. 7. In particular, we choose two extreme possibilities, which are 
motivated both with model available in literature and with an observational point of view.

The first has $Q_{H I I}(z=10)=0.2$. This assumption agrees with the $68 \%$ credibility interval modelled on the marginalized distribution of the neutral fraction $\left(1-Q_{H I I}\right)$, from the SFR histories and the Planck constraints on $\tau_{e s}$, from Robertson et al. (2015) [52]. Similarly, it is coherent with the range of $Q_{H I I}$ allowed for the model by Bouwens et al. (2015), where Reionization is complete between $z=5.9$ and $z=6.5$ [36].

As a second possibility, we choose $Q_{H I}(z=9)=0.0$, which is preferred by the two hydrogen neutral fraction measurements performed by Mason et al. (2019) and Hoag et al. (2018) and discussed in sec. 4.1 [8,9]. All the others are intermediate cases.

4.3. Reionization with galaxies only

4.3.1. Implications on $f_{\text {esc }}$

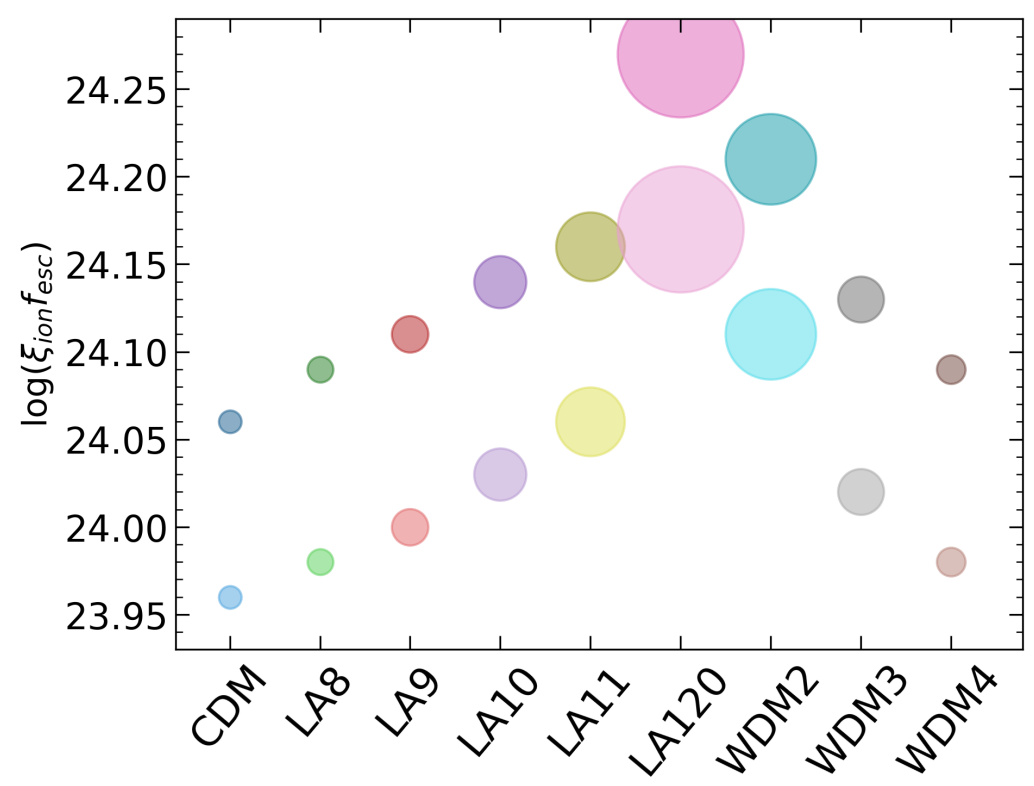

Figure 7. The panel shows the values of the product $\xi_{\text {ion }} f_{\text {esc }}$ needed to ionize the IGM at $(z=6.7)$, for a set of different cosmologies. The dot size increases with $M_{h m}$; lighter colours refer to initial condition $Q_{H I I}(z=10)=0.2$, while darker colours are for $Q_{H I I}(z=9)=0.0$.

For each of the two initial conditions we compute the number density of ionizing photons $\dot{N}_{i o n}$ with different combination of $\xi_{\text {ion }} f_{\text {esc }}$, exploring the effect of the parameters degeneracy on the reheating of IGM.

Models that are affected by a stronger free-streaming have an higher $M_{h m}$ value, which has consequence on galaxy formation, determining a lack of faint-galaxies which alters the UV LF (see Fig. 1), with a general reduction in the UV luminosity density. Thus, we obtain a delay in the IGM ionizing process, with respect to CDM.

In Fig. 7, we show $\log \left(\xi_{\text {ion }} f_{\text {esc }}\right)$ in CDM, sterile neutrinos and thermal WDM cosmologies. Because of the great uncertainty on $f_{\text {esc }}$, we searched for the $\xi_{\text {ion }} f_{\text {esc }}$ values which ensure the completion of Reionization by $z=6.7$. We note that $\log \left(\xi_{\text {ion }} f_{\text {esc }}\right)$ increases with $M_{h m}$ : a greater escape fraction and/or UV photons production efficiency is needed to complete the Reionization process in WDM scenarios.

The quantity $\xi_{\text {ion }}$ is however better constrained than $f_{\text {esc }}$, so we assume from the literature a fiducial value of $\log \left(\xi_{\text {ion }}\right)=25.2[31,36,53,54]$, as expected from a low metallicity single stars population. We did not investigate the variation of $\xi_{\text {ion }}$ with redshift and $M_{U V}$, which we have considered negligible with respect to changing in escape fraction. This hypotheses allows us to set an upper limit to $f_{\text {esc }}$ for any different WDM particle and boundary condition. In general, models that start from $Q=0$ need an higher $f_{\text {esc }}$ value 
to ionize the IGM within the same $z$ range. For this reason they are more inclusive and result in a weaker constraints to the admitted escape fraction. If $f_{\text {esc }}>f_{\text {esc }}^{\text {sup }}$, Reionization process is completed outside the fiducial redshift interval.

In table 4 we summarize our results for the two initial condition discussed in sec. 4.2.

Table 4. Product $\log \left(\xi_{\text {ion }} f_{\text {esc }}\right)$ and upper limit of $f_{\text {esc }}$, with $\xi_{\text {ion }}=25.2$, for different cosmologies and different initial conditions. We note that these two quantities increase with the half-mode mass $\left(M_{h m}\right)$. The case [1] refers to $Q_{H I I}(z=10)=0.2$. The case [2] refers to $Q_{H I I}(z=9)=0.0$.

\begin{tabular}{cccccc}
\hline Name & $M_{h m}\left(M_{\odot}\right)$ & $\log \left(\xi_{\text {ion }} f_{\text {esc }}\right)[1]$ & $f_{\text {esc,sup }}[1]$ & $\log \left(\xi_{\text {ion }} f_{\text {esc }}\right)[2]$ & $f_{\text {esc,sup }}[2]$ \\
\hline CDM & - & 23.96 & 0.06 & 24.06 & 0.07 \\
LA8 & $1.3 \times 10^{8}$ & 23.98 & 0.06 & 24.09 & 0.08 \\
LA9 & $2.6 \times 10^{8}$ & 24.00 & 0.06 & 24.11 & 0.08 \\
LA10 & $5.3 \times 10^{8}$ & 24.03 & 0.07 & 24.14 & 0.09 \\
LA11 & $9.2 \times 10^{8}$ & 24.06 & 0.07 & 24.16 & 0.09 \\
LA120 & $3.1 \times 10^{9}$ & 24.17 & 0.09 & 24.27 & 0.12 \\
WDM 2 & $1.6 \times 10^{9}$ & 24.11 & 0.08 & 24.21 & 0.10 \\
WDM 3 & $4.1 \times 10^{8}$ & 24.02 & 0.07 & 24.13 & 0.08 \\
WDM 4 & $1.6 \times 10^{8}$ & 23.98 & 0.06 & 24.09 & 0.08 \\
\hline
\end{tabular}

\subsubsection{Implications on the Reionization history}

In our reconstruction of EoR, we found that the bulk of Reionization happens between $6<z<8$, with significant differences which derive from initial condition and $f_{\text {esc }}$ model.

In fig. 8 we show the evolution of the filling fraction in sterile neutrino and thermal WDM scenarios, with $\log \left(\xi_{\text {ion }}\right)=25.2$. We note that the Reionization pattern changes with respect to the initial condition. Models with $Q_{H I I}(z=9)=0.0$, which have $f_{\text {esc }}=0.06$ in the plot, show a better agreement with the data, not only with respect to Hoag19 and Mason19 measurements, but also with lower redshift observational points. Differences between cosmologies are tiny, but increase with the age of universe. However, they do not allow us to discriminate between CDM and WDM scenarios with the actual experimental uncertainties.

The initial condition $Q_{H I I}(z=10)=0.2$ requires a lower escape fraction $\left(f_{\text {esc }}=0.05\right)$, because the IGM is already partial ionized when we start to solve eq. 7 .

The model by Yajima et al. (2011) leads to a more linear evolution of $Q_{H I I}$ with redshift: the contribution of bright galaxies is strongly suppressed, so they cannot imprint the characteristic acceleration that we see in fixed $f_{\text {esc }}$ models.

In table 2 we show the changes in $f_{e s c}$, which reaches even the $32 \%$ for less massive halos. An higher escape fraction requires at the same time to reduce $\xi_{\text {ion }}$. For this reason, the case with $Q_{H I I}=0.2$ is strongly disfavoured from both the data and the $\operatorname{low} \log \left(\xi_{\text {ion }}\right)=24.9$ value. From fig 9 , if we take $Q_{H I I}(z=9)=0$, we obtain a better agreement, and $\log \left(\xi_{\text {ion }}\right)$ can increase to 25.0 .

Finally, the analysis of electron scattering optical depth shows a $1 \sigma$ agreement with the Planck 2015 result. In particular, if $Q_{H I I}(z=9)=0, \tau_{e s}$ saturates in most models within the range of Planck 2018 measurements. Conversely, we should note that cosmological scenarios with $Q_{H I I}(z=10)=0.2$ cannot reach their definitive optical depth value, which depends on how the $Q_{H I I}(z)$ decreases at $z>10$. Further improvements in our semi-analytic model will allow us to repeat the study at previous redshifts. 

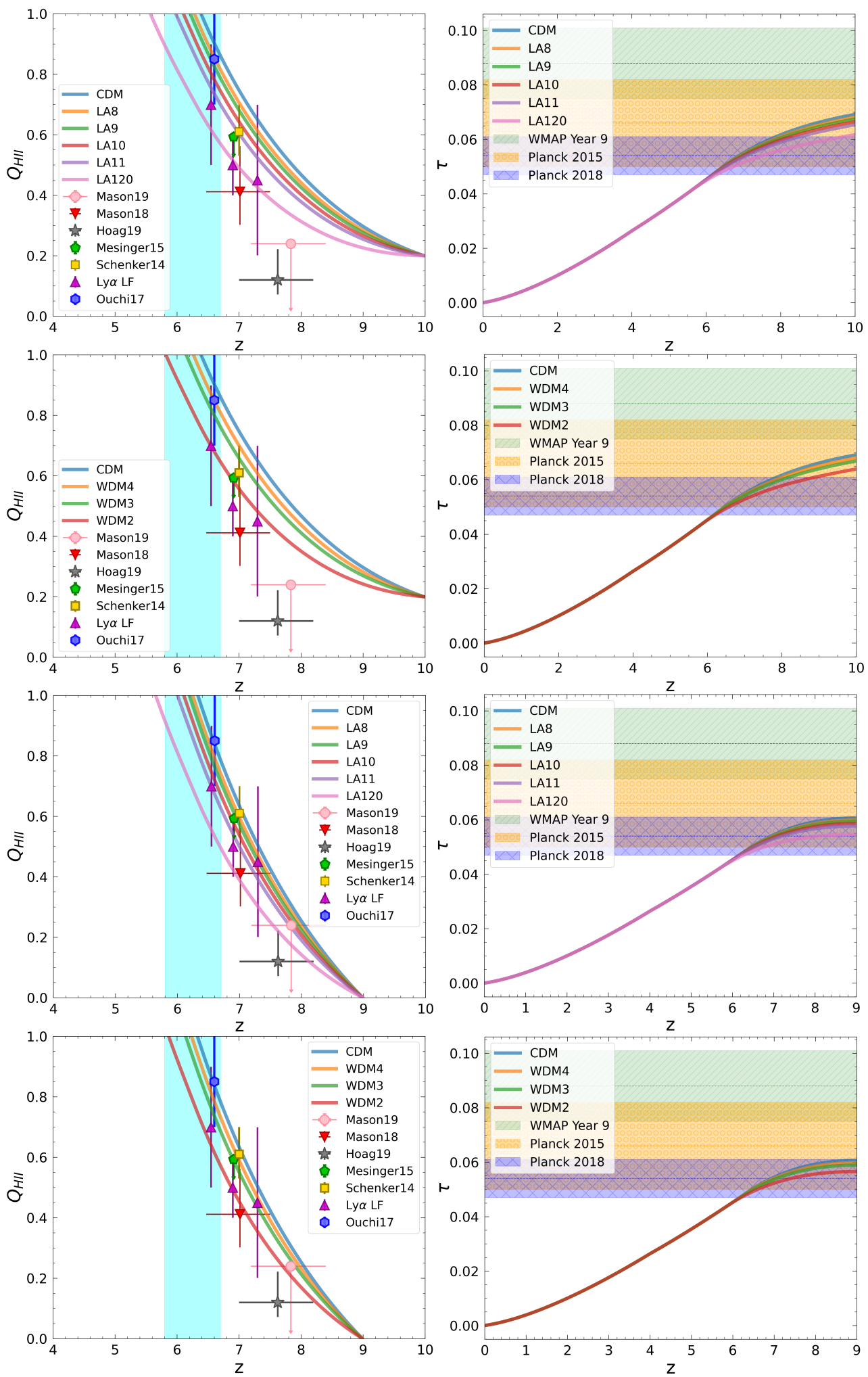

Figure 8. (a) Left: evolution of the filling fraction $Q_{H I I}$, for sterile neutrino and thermal WDM models, with $\log \left(\xi_{\text {ion }}\right)=25.2$. The two upper panels have initial condition $Q_{H I I}(z=10)=0.2$ and $f_{e s c}=0.05$. The two lower panels are plotted with $Q_{H I I}(z=$ $9)=0.0$ and $f_{e s c}=0.06$. The cyan shaded region indicates our fiducial late-Reionization redshift interval, $5.8<z<6.7$. The upward triangle labeled Ly $\alpha$ LF includes results by Konno et al. (2014), Konno et al. (2017) and Zheng et al. (2017) [46-48]. (b) Right: electron scattering optical depth for different models, compared with measure from Planck and WMAP [1-3]. 

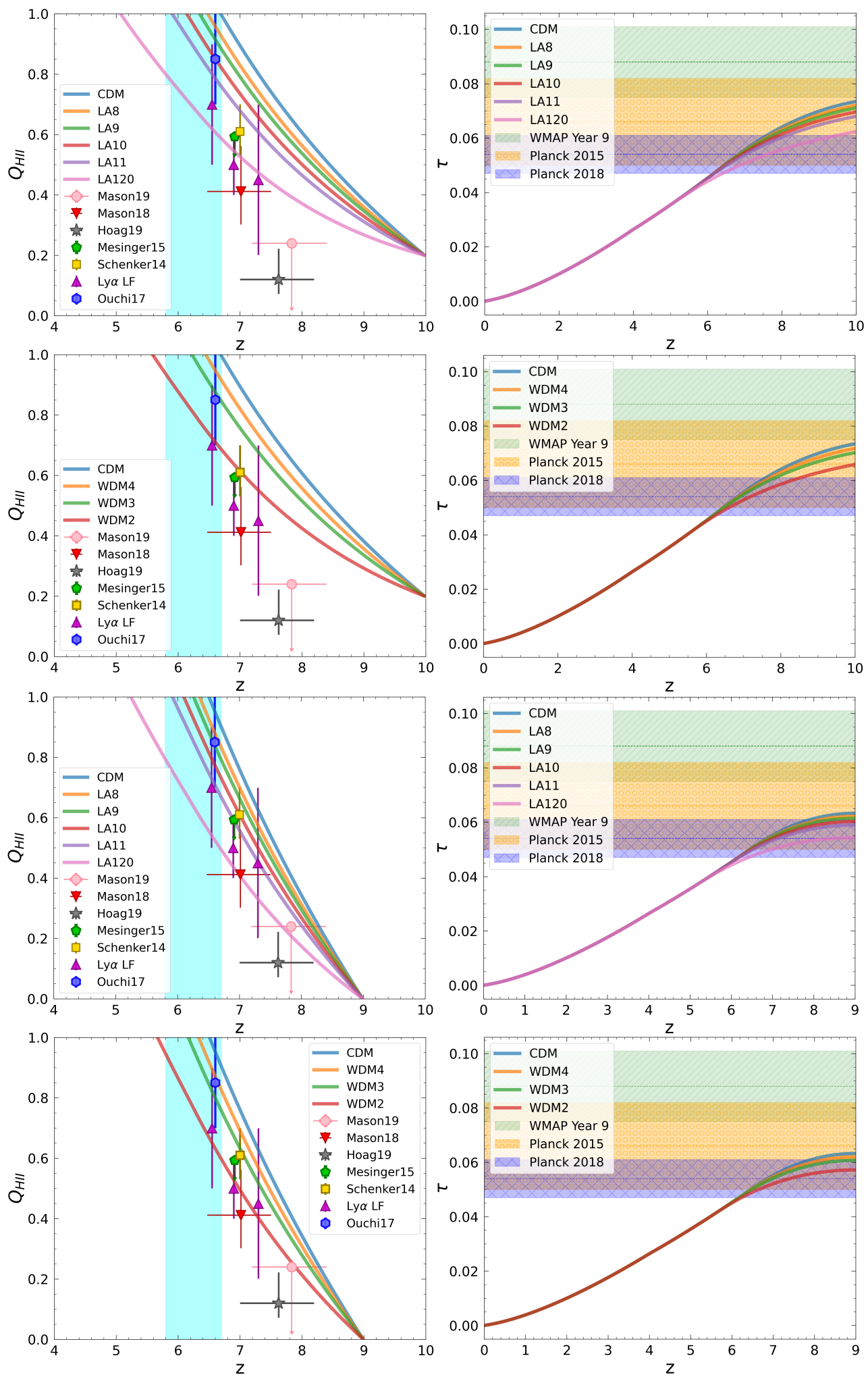

Figure 9. (a) Left: evolution of the filling fraction $Q_{H I I}$, for sterile neutrino and thermal WDM models, with $f_{\text {esc }}\left(M_{\text {halo }}\right)$ as computed by Yajima et al.(2011) [32]. The two upper panels have initial condition $Q_{H I I}(z=10)=0.2$ and $\log \left(\xi_{\text {ion }}\right)=24.9$. The two lower panels are plotted with $Q_{H I I}(z=9)=0.0$ and $\log \left(\xi_{\text {ion }}\right)=25.0$. The cyan shaded region indicates our fiducial late-Reionization redshift interval, $5.8<z<6$.7. The upward triangle labeled Ly $\alpha$ LF includes results by Konno et al. (2014), Konno et al. (2017) and Zheng et al. (2017). [46-48] (b) Right: electron scattering optical depth for different models, compared with measure from Planck and WMAP [1-3]. 


\subsection{Reionization with galaxies and AGN}

4.4.1. Dependence on spectral index

In fig. 10 we illustrate the evolution of the filling fraction with UV photons from both galaxies and AGNs. The plot refers to CDM cosmology and allows to analyse the impact of $\alpha_{U V}$ on Reionization history.

In general, the harder is the spectral index, the faster is the Reionization process; in the range $[-2.31,-1.09]$, a $\Delta z \approx 0.2$ maximum difference is admitted. Furthermore, this effect overlaps with the choice of initial condition. Indeed, an higher initial HII filling fraction is partially erased by the variation in the power-law slope. We therefore conclude that the UV spectral slope gives only a perturbative contribution to Reionization history. This happens because the role of the AGNs UV continuum is minor, with respect to the their importance in the escape fraction production mechanism.

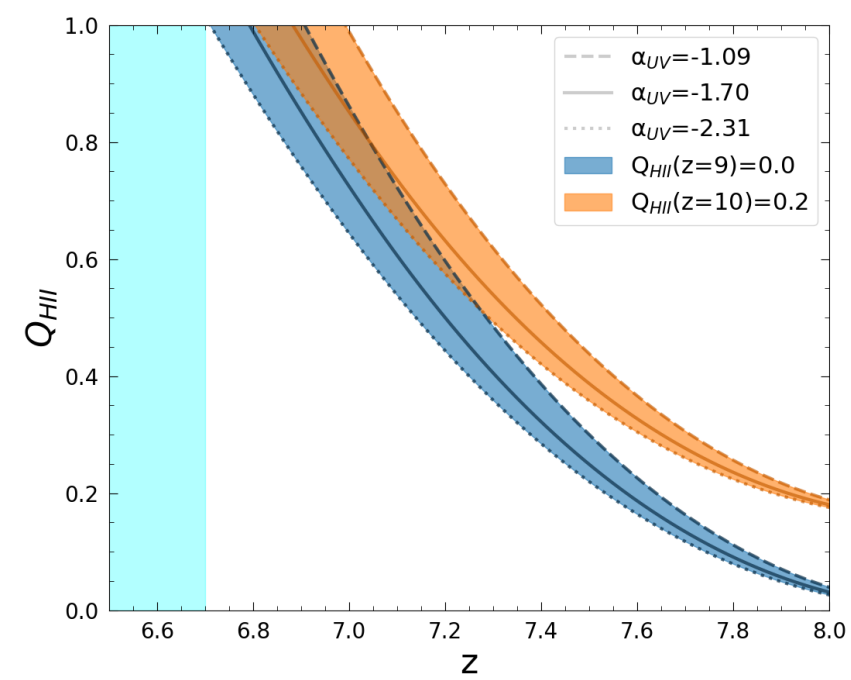

Figure 10. The panel shows the dependence of Reionization history on the power-law slope $\alpha_{U V}$, for the CDM cosmology: the continuous line refers to the central value $\alpha_{U V}=-1.70$, while the dotted and the dashed lines indicate respectively the lower and the upper limit $(-2.31$ and -1.09$)$ of the slope derived by Lusso et al. (2015) [42].
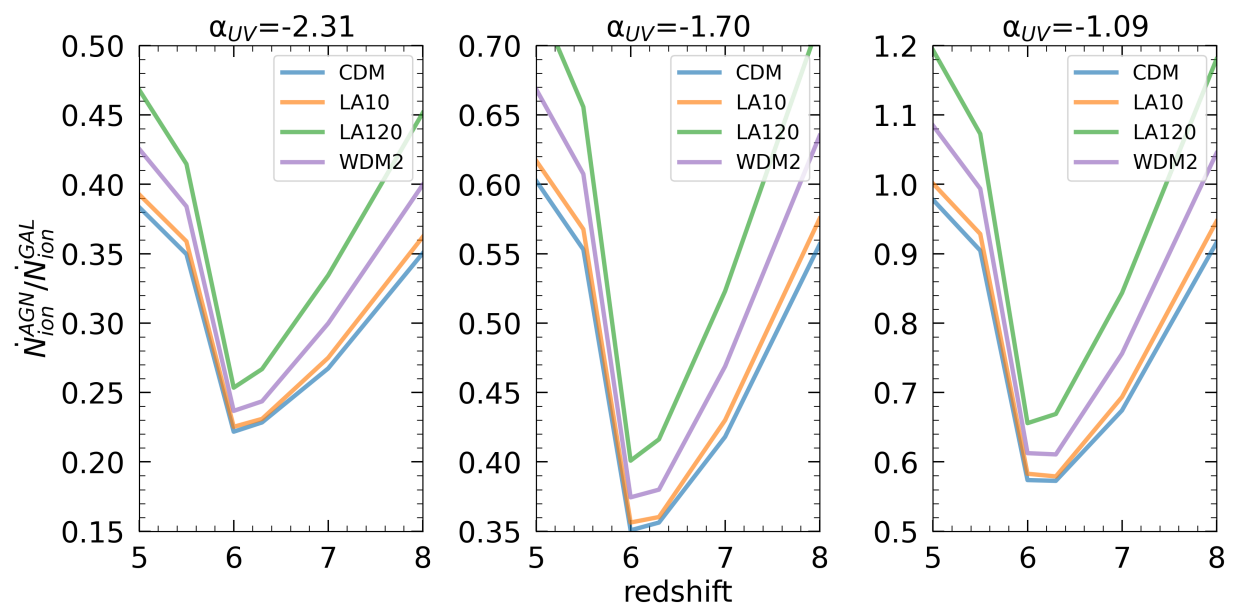

Figure 11. Evolution of $\frac{\dot{N}_{i o n}^{A G N}}{\hat{N}_{i o n}^{G A L}}$ with $z$, for $\alpha_{U V}=-1.70 \pm 0.61$, as derived by Lusso et al. (2015) [42]. We represent CDM, LA10, LA120 and WDM2 cosmologies: all the others are intermediate cases.

Fig. 11 describes some relevant features which emerge from the evolution, with $z$ and $\alpha_{U V}$, of $\dot{N}_{i o n}^{A G N} / \dot{N}_{i o n}^{G A L}$ in some representative cosmological models. The role of 
accretion increases at $z<6$, but decreases between $6<z<8$, as the bright galaxies emission becomes more important. Changes in the UV spectral shape have consequences on the relative importance of AGN photons density, which depends on the $\xi_{i o n}^{A G N}$ distribution. Despite to this variations, the quantity $\dot{N}_{i o n}^{A G N} / \dot{N}_{i o n}^{G A L}$ remains, in CDM case, less than unity for any redshift and any considered $\alpha_{U V}$ value. Our $\alpha_{U V}=-1.09$ result partially agrees with Finkelstein et al. (2019), which develop a "galaxy-dominated" model until $z<4.6$ and with Dayal et al. (2020), in which the equivalence between star formation and accretion contributes is reached only at $z \approx 5[31,55]$. We also note that the $\dot{N}_{i o n}^{A G N} / \dot{N}_{i o n}^{G A L}$ ratio increases in WDM cosmologies, where the AGN energy production is not altered. Because of free-streaming, its growth is more pronounced in model with an higher half-mode mass and a more suppressed faint-galaxies emission. Nevertheless, excluding LA120 and WDM2 scenarios for $z=8$, galaxies are still the dominant source during EoR.

\subsubsection{Implications on the Reionization history}

Fig. 12 summarizes our results, obtained counting the contribution of both galaxies and AGNs. The bulk of Reionization still happens between $7<z<8$, but interestingly, the blast-wave model reduces the dependence from initial conditions; in our high-z extrapolation of the UV LFs, the lack of a sufficient ionizing background causes a partial IGM recombination, which induces a good fit with Mason19 observation. This happens because, the AGN-induced $f_{\text {esc }}$ surpasses the importance of objects with no accretion process and selects the ones with an higher accretion luminosity. Thus, it favours the impact of active bright-galaxies, whose number density strongly decreases at high redshift. The evolution of the filling fraction in fig. 12 is plotted with $\alpha_{U V}=-2.31$. However, we have already shown that the AGN UV power-law slope does not affect too much the Reionization pattern, and its changing partially overlaps with the choice of the initial condition. In any case, fig. 10 reveals that the IGM is completely ionized at $z<7$. The analysis of electron scattering optical depth is in agreement with Planck 2015 measurements. Furthermore, if we consider $Q_{H I I}(z=9)=0$, which represents the saturated case, the $\tau_{e s}$ value falls within the $1 \sigma$ Planck 2018 interval.

The AGN blast-wave model thus provides a very interesting tool to retrace the Reionization history during the cosmic time, which is also very robust against changing in the accretion spectral shape. However, due to the minor role of faint-galaxies UV photons, this model is less dependent from cosmology. Indeed, it introduces a degeneracy in the evolution of $Q_{H I I}(z)$ and $\tau_{e s}$ between CDM and WDM scenarios (with the exception of LA120 and WDM2), which makes impossible to exploit macroscopic observations of Reionization history to discriminate between $\Lambda$ CDM model and other alternative cosmologies. 

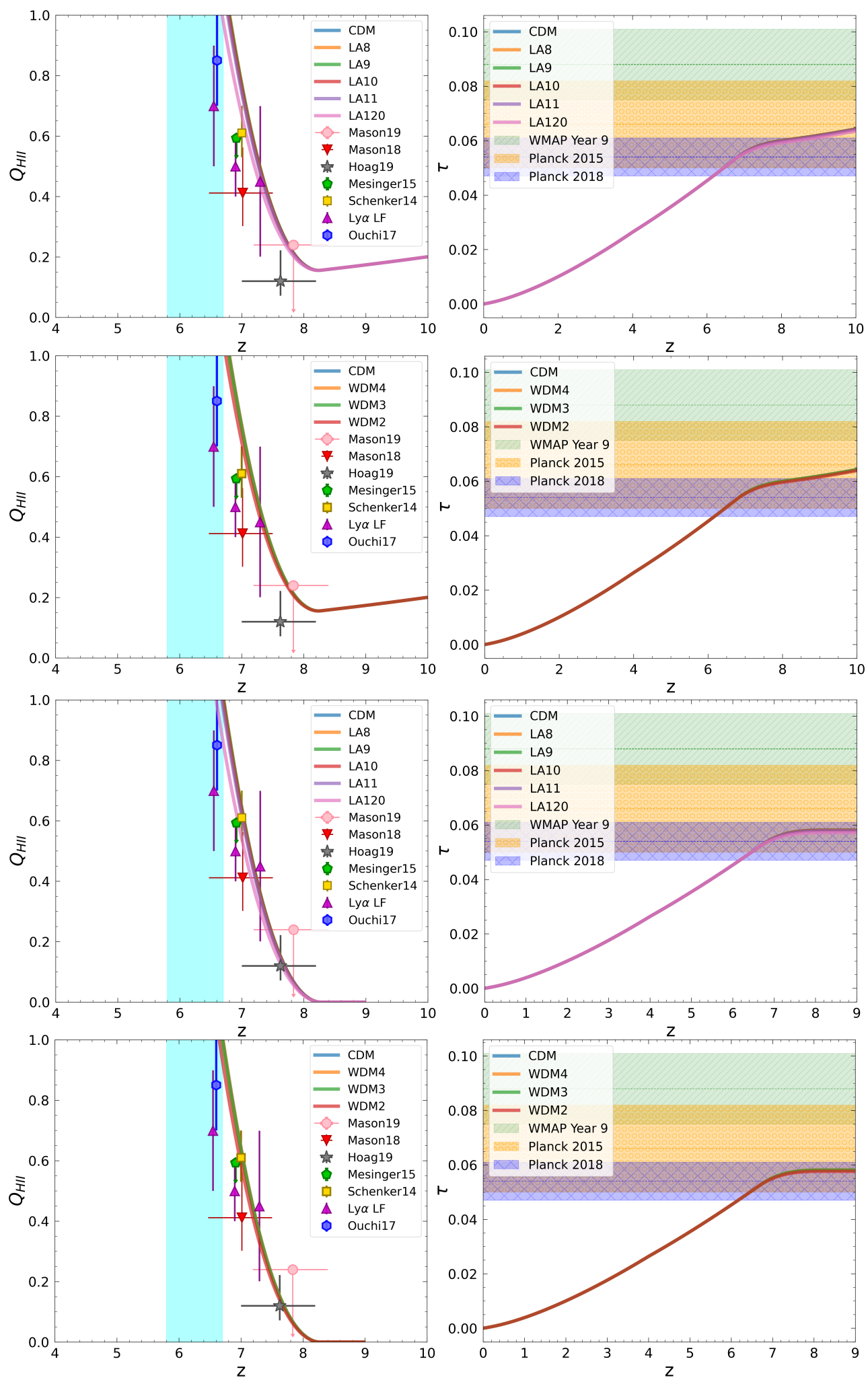

Figure 12. (a) Left: evolution of the filling fraction $Q_{H I I}$, for sterile neutrino and thermal WDM models, where $f_{\text {esc }}$ is obtained with the AGN blast-wave model. The two upper panels have initial condition $Q_{H I}(z=10)=0.2$, the two lower panels are plotted with $Q_{H I I}(z=9)=0.0$. We use $\log \left(\xi_{i o n}^{g a l}\right)=25.2$, while $\xi_{i o n}^{A G N}$ is computed with $\alpha_{U V}=-2.31$ spectral slope, according to Lusso et al. (2015) [42]. The cyan shaded region indicates our fiducial late-Reionization redshift interval, $5.8<z<6$.7. The upward triangle labeled Ly $\alpha$ LF includes results by Konno et al. (2014), Konno et al. (2017) and Zheng et al. (2017). [46-48] (b) Right: electron scattering optical depth for different models, compared with measure from Planck and WMAP [1-3]. 


\subsection{Comparison with previous works on Reionization}

In this work, we have used different assumptions about the escape fraction value. In the hypothesis that galaxies are the only ionizing source during the EoR, we find an upper limit to $f_{\text {esc }}$ for each considered cosmology. Depending on initial condition, it can varies between 0.06 and 0.12 . However, $f_{\text {esc }}$ is a very low-constrained parameter in literature; it depends from a huge number of physical properties and there are many studies that offers their proper estimation (see Dayal P. and Ferrara A. for a review [56]). Conversely, our AGN $f_{e s c}$ approaches the observational results presented by Grazian et al. (2018) [44], which offer a mean value of $74 \%$.

Concerning Reionization history, we conclude that, in our blast-wave model, the IGM is almost ionized at $z \approx 6.7$, with a weak dependency on $\alpha_{U V}$. It is interesting to discuss this result in the context of previous studies about the EoR. Dayal et al. (2020) found an end redshift of Reionization between $5<z<6.5$, analyzing both the role of galaxies and AGNs [55]. Finkelstein et al. (2019) completed the Reionization at $z \approx 6$, using halo-mass dependent low escape fractions [31]. In these two galaxy-driven works, AGNs become dominant at $z \lesssim 5$. Moreover, Bouwens et al. (2015) concluded that $Q_{H I I}$ reaches the unity between $5.9<z<6.5$ [36], while Robertson et al. (2015), using $f_{\text {esc }}=0.2$, highlighted an evolution of the filling fraction from 0.2 to 0.9 during $6<z<9$ [52], with $\tau_{\text {esc }}$ within the Planck $20151 \sigma$ range result. Finally, we remind the work by Dayal et al. (2017), which suggest that, in CDM case, the bulk of the ionizing photons production is related to low-mass systems with $-15<M_{U V}<-10$, while in 1.5 and $3 \mathrm{keV}$ thermal WDM it is shifted towards $-17<M_{U V}<-13$ [21]. This result is slightly different from our galaxies-only Reionization model, which instead admits a significant contribution from $M_{U V} \gtrsim-20$ sources.

\section{Summary and conclusions}

In this paper we have studied how the suppression of the CDM power spectrum due to particles free streaming can have macroscopic consequences, via a late galaxy formation, on the Reionization of IGM. We have used the semi-analytic model described in Menci et al. (2018) [20], to produce the UV LF in a $\Lambda$ CDM framework. Suppressing the contribution of the faint galaxies, we have tested some $\Lambda \mathrm{WDM}$ cosmologies: in particular, we have focused on five sterile neutrino models (LA8-LA9-LA10-LA11-LA120, presented in Lovell et al. (2020) [26]), with the same particle mass, but with different mixing angle, and three thermal WDM models (WDM2-WDM3 and WDM4), with respectively $m_{X}=2-3-4 \mathrm{keV}$. In both cases, we have checked that an higher $M_{h m}$ leads to a general delay in the Reionization process.

First of all, we have explored the hypothesis that Reionization is driven by galactic photons only:

- if $f_{e s c}$ is fixed, in CDM case, we find that merging between halos determines the rise of the $M_{U V}<-20$ systems relative contribution to the ionizing photons budget, from $\approx 30 \%$ to $\approx 45 \%$ between $6.3<z<8$. However, it remains subdominant during the EoR, because faint galaxies with $M_{U V}>-20$ emit the bulk of ionizing photons. On the other hand, in WDM scenarios, the particles free-streaming yields to a shift towards brighter sources. In particular, $r_{\text {phot }}\left(M_{U V}<-20\right)$ undergoes a further $1-10 \%$ growth, depending on cosmology.

- if we use the $f_{e s c}\left(M_{\text {halo }}\right)$ model by Yajima et al. (2011) [32], the impact of $M_{U V}<$ -20 systems remains approximately constant during EoR, with a value that goes from $15 \%(\mathrm{CDM})$ to $25 \%$ (LA120). We also note that faint galaxies with $-14<$ $M_{U V}<-12$ provides up to $4 \%$ of $\dot{N}_{\text {ion }}$ in CDM case.

In general, WDM scenarios yield to an overall reduction of $\dot{N}_{\text {ion }}$ with respect to CDM. The Reionization history depends also on the choices of the initial ionized hydrogen filling fraction: 
- $\quad$ in fixed $f_{\text {esc }}$ models, the completion of Reionization process at $z<6.7$, requires an increase in the $f_{\text {esc }} \xi_{\text {ion }}$ product with $M_{h m}$. Using $\log \left(\xi_{\text {ion }}\right)=25.2$ we set an upper limit $f_{\text {esc }}^{\text {sup }}$ that goes, depending on the high-z $Q_{H I I}$ value, from 0.06 in the $\Lambda C D M$ model to 0.12 in the LA120 cosmology;

- $\quad$ models with $Q_{H I I}(z=9)=0$ better agree with observational data, while models with $Q_{H I I}(z=10)=0.2$ do not match the neutral fraction measurements performed by Mason et al. (2019) and Hoag et al. (2019) [8,9];

- the evolution of $Q_{H I I}$ with escape fraction by Yajima et al. (2011) is strongly dependent from the choice of the initial condition and it is also disfavoured by the low $\log \left(\xi_{\text {ion }}\right) \approx 24.9-25.0$ value.

We then have implemented the AGN blast-wave model developed by Menci et al. (2019) [38], which explain the genesis of the ionizing photons escape fraction in the context of feedback and co-evolution between Active Galactic Nuclei and host galaxies. In particular:

- we find a correlation between $f_{e s c}$ and $L_{b o l}^{A G N}$ and a bimodality in the escape fraction distribution. The majority of systems have $f_{\text {esc }} \approx 0$ or $f_{\text {esc }} \approx 0.7$, so the blastwave model promotes the contribution the brightest ones, which contain the most luminous AGNs;

- the $\xi_{i o n}^{A G N}$ distribution depends on the black-body accretion temperature and on the UV AGNs spectral slope, which values $\alpha_{U V}=-1.70 \pm 0.61$ from observational constraints by Lusso et al. (2015) [42];

- changes in $\alpha_{U V}$ do not alter significantly the Reionization history. For a CDM cosmology, the redshift at which hydrogen is fully ionized can vary within a range of at most $\Delta z \approx 0.2$. Furthermore, this effect partially overlaps with differences in initial conditions;

- the $\dot{N}_{i o n}^{A G N} / \dot{N}_{i o n}^{\text {gal }}$ ratio is constantly below unity, except for WMD2 and LA120 if $\alpha=$ -1.09 . This result suggests that, while AGNs are important for the $f_{\text {esc }}$ production mechanism, their emission plays just a secondary role during the EoR;

- finally, the blast-wave model provides a good agreement with high-z $Q_{H I I}$ observations and completes the reheating of IGM at $z<7$. It also reduces the dependence on initial conditions, but introduces a degeneracy in $Q_{H I I}$ and $\tau_{e s}$ between different cosmological scenarios, that make impossible to discriminate between CDM and less suppressed WDM cosmologies.

To conclude, we note that the most important limits to our analysis are related to observational uncertainties. In the future, we expect significant advances in the field of extra-galactic astrophysics and observational cosmology. For example, the role of AGNs during EoR will be better understood with Euclid, by looking at the Quasar Luminosity Function in the early universe. Also the detection of QSOs damping wing can be used to measure the cosmic neutral hydrogen fraction [57]. Although the electron scattering optical depth and the neutral hydrogen fraction provide important constraints on Reionization redshift, further spectroscopic measurements are needed in order to characterize high-z galaxies $f_{\text {esc }}$ and $\xi_{\text {ion }}$. Moreover, a better estimation of the $z>6 \mathrm{UV}$ LF will allow to search for a possible turn-over in its faint-end, and to set constraints on WDM cosmological scenarios. Both goals can be achieved by exploring the deep parts of the universe that will be accessible to the James Webb Space Telescope.

Author Contributions: Conceptualization, N.M., M.C.; methodology, M.R, N.M. and M.C; software, N.M.; validation, N.M., M.C.; data curation, M.R.; writing-original draft preparation, M.R.; writing-review and editing, M.R., N.M and M.C.; visualization, M.R.; supervision, N.M., M.C.; project administration, N.M., M.C. All authors have read and agreed to the published version of the manuscript.

Funding: This research received no external funding 
Acknowledgments: We thank N. Sanchez for inviting us to write the present paper, L. Pentericci for supervision during the work and M. Parente for the aesthetic advice.

Conflicts of Interest: The authors declare no conflict of interest.

\section{References}

1. Planck Collaboration. Planck 2015 results - XIII. Cosmological parameters. AEA 2016, 594, A13. doi:10.1051/0004-6361/201525830.

2. Planck Collaboration. Planck 2018 results - VI. Cosmological parameters. AEA 2020, 641, A6. doi:10.1051/0004-6361/201833910.

3. Hinshaw, G.; Larson, D.; Komatsu, E.; Spergel, D.N.; Bennett, C.L.; Dunkley, J.; Nolta, M.R.; Halpern, M.; Hill, R.S.; Odegard, N.; Page, L.; Smith, K.M.; Weiland, J.L.; Gold, B.; Jarosik, N.; Kogut, A.; Limon, M.; Meyer, S.S.; Tucker, G.S.; Wollack, E.; Wright, E.L. Nine-Year Wilkinson Microwave Anisotropy Probe (WMAP) Observations: Cosmological Parameter Results. The Astrophysical Journal Supplement Series 2013, 208, 19. doi:10.1088/0067-0049/208/2/19.

4. Fan, X.; Carilli, C.; Keating, B. Observational Constraints on Cosmic Reionization. Annual Review of Astronomy and Astrophysics 2006, 44, 415-462. doi:10.1146/annurev.astro.44.051905.092514.

5. Wise, J.H. Cosmic reionisation. Contemporary Physics 2019, 60, 145-163. doi: 10.1080/00107514.2019.1631548.

6. Dijkstra, M. Ly $\alpha$ Emitting Galaxies as a Probe of Reionisation. Publications of the Astronomical Society of Australia 2014, 31. doi:10.1017/pasa.2014.33.

7. Pentericci, L.; Vanzella, E.; Fontana, A.; Castellano, M.; Treu, T.; Mesinger, A.; Dijkstra, M.; Grazian, A.; Bradač, M.; Conselice, C.; Cristiani, S.; Dunlop, J.; Galametz, A.; Giavalisco, M.; Giallongo, E.; Koekemoer, A.; McLure, R.; Maiolino, R.; Paris, D.; Santini, P. New Observations of $\mathrm{z} \sim 7$ Galaxies: Evidence for a Patchy Reionization. The Astrophysical Journal 2014, 793, 113. doi:10.1088/0004-637x/793/2/113.

8. Hoag, A.; Bradač, M.; Huang, K.; Mason, C.; Treu, T.; Schmidt, K.B.; Trenti, M.; Strait, V.; Lemaux, B.C.; Finney, E.Q.; Paddock, M. Constraining the Neutral Fraction of Hydrogen in the IGM at Redshift 7.5. The Astrophysical Journal 2019, 878, 12. doi:10.3847/15384357/ab1de7.

9. Mason, C.A.; Fontana, A.; Treu, T.; Schmidt, K.B.; Hoag, A.; Abramson, L.; Amorin, R.; Bradač, M.; Guaita, L.; Jones, T.; Henry, A.; Malkan, M.A.; Pentericci, L.; Trenti, M.; Vanzella, E. Inferences on the timeline of reionization at $\mathrm{z} \sim 8$ from the KMOS Lens-Amplified Spectroscopic Survey. Monthly Notices of the Royal Astronomical Society 2019, 485, 3947-3969. doi:10.1093/mnras/stz632.

10. Bullock, J.S.; Boylan-Kolchin, M. Small-Scale Challenges to the CDM Paradigm. Annual Review of Astronomy and Astrophysics 2017, 55, 343-387. doi:10.1146/annurev-astro-091916055313.

11. Klypin, A.; Kravtsov, A.V.; Valenzuela, O.; Prada, F. Where Are the Missing Galactic Satellites? The Astrophysical Journal 1999, 522, 82-92. doi:10.1086/307643.

12. Yue, B.; Castellano, M.; Ferrara, A.; Fontana, A.; Merlin, E.; Amorín, R.; Grazian, A.; MármolQueralto, E.; Michałowski, M.J.; Mortlock, A.; Paris, D.; Parsa, S.; Pilo, S.; Santini, P.; Criscienzo, M.D. On the Faint End of the Galaxy Luminosity Function in the Epoch of Reionization: Updated Constraints from the HST Frontier Fields. The Astrophysical Journal 2018, 868, 115. doi:10.3847/1538-4357/aae77f.

13. Menci, N.; Grazian, A.; Castellano, M.; Sanchez, N.G. A Stringent Limit on the Warm Dark Matter Particle Masses From the Abundance of $z=6$ Galaxies in the Hubble Frontier Fields. The Astrophysical Journal 2016, 825, L1. doi:10.3847/2041-8205/825/1/11.

14. Dayal, P.; Mesinger, A.; Pacucci, F. Early Galaxy Formation in Warm Dark Matter Cosmologies. The Astrophysical Journal 2015, 806, 67. doi:10.1088/0004-637x/806/1/67.

15. Castellano, M.; Yue, B.; Ferrara, A.; Merlin, E.; Fontana, A.; Amorín, R.; Grazian, A.; MármolQueralto, E.; Michałowski, M.J.; Mortlock, A.; Paris, D.; Parsa, S.; Pilo, S.; Santini, P. Constraints on Photoionization Feedback from Number Counts of Ultra-Faint High-Redshift Galaxies in the Frontier Fields. The Astrophysical Journal 2016, 823, L40. doi:10.3847/20418205/823/2/140.

16. Corasaniti, P.; Agarwal, S.; Marsh, D.; Das, S. Constraints on dark matter scenarios from measurements of the galaxy luminosity function at high redshifts. Physical Review D 2017, 95. doi:10.1103/physrevd.95.083512. 
17. Viel, M.; Becker, G.D.; Bolton, J.S.; Haehnelt, M.G. Warm dark matter as a solution to the small scale crisis: New constraints from high redshift Lyman- $\alpha$ forest data. Phys. Rev. D 2013, 88, 043502. doi:10.1103/PhysRevD.88.043502.

18. Merle, A. keV sterile neutrino Dark Matter, 2017, [arXiv:hep-ph/1702.08430].

19. Iakubovskyi, D. Observation of the new emission line at $3.5 \mathrm{keV}$ in X-ray spectra of galaxies and galaxy clusters. Advances in Astronomy and Space Physics 2016, 6, 3-15. doi:10.17721/22271481.6.3-15.

20. Menci, N.; Grazian, A.; Lamastra, A.; Calura, F.; Castellano, M.; Santini, P. Galaxy Formation in Sterile Neutrino Dark Matter Models. The Astrophysical Journal 2018, 854, 1. doi: 10.3847/1538-4357/aaa773.

21. Dayal, P.; Choudhury, T.R.; Bromm, V.; Pacucci, F. Reionization and Galaxy Formation in Warm Dark Matter Cosmologies. The Astrophysical Journal 2017, 836, 16. doi:10.3847/15384357/836/1/16.

22. Lovell, M.R.; Bose, S.; Boyarsky, A.; Cole, S.; Frenk, C.S.; Gonzalez-Perez, V.; Kennedy, R.; Ruchayskiy, O.; Smith, A. Satellite galaxies in semi-analytic models of galaxy formation with sterile neutrino dark matter. Monthly Notices of the Royal Astronomical Society 2016, 461, 60-72. doi:10.1093/mnras/stw1317.

23. Bode, P.; Ostriker, J.P.; Turok, N. Halo Formation in Warm Dark Matter Models. The Astrophysical Journal 2001, 556, 93-107. doi:10.1086/321541.

24. Schneider, A.; Smith, R.E.; Macciò, A.V.; Moore, B. Non-linear evolution of cosmological structures in warm dark matter models. Monthly Notices of the Royal Astronomical Society 2012, 424, 684-698. doi:10.1111/j.1365-2966.2012.21252.x.

25. Viel, M.; Lesgourgues, J.; Haehnelt, M.G.; Matarrese, S.; Riotto, A. Constraining warm dark matter candidates including sterile neutrinos and light gravitinos with WMAP and the Lyman- $\alpha$ forest. Phys. Rev. D 2005, 71, 063534. doi:10.1103/PhysRevD.71.063534.

26. Lovell, M.R. Toward a General Parameterization of the Warm Dark Matter Halo Mass Function. The Astrophysical Journal 2020, 897, 147. doi:10.3847/1538-4357/ab982a.

27. Bouwens, R.J.; Illingworth, G.D.; Oesch, P.A.; Trenti, M.; Labbé, I.; Bradley, L.; Carollo, M.; van Dokkum, P.G.; Gonzalez, V.; Holwerda, B.; Franx, M.; Spitler, L.; Smit, R.; Magee, D. UV Luminosity Functions at Redshifts $\mathrm{Z} \sim 4$ to $\mathrm{Z} \sim 10: 10,000$ Galaxies from HST Legacy Fields. The Astrophysical Journal 2015, 803, 34. doi:10.1088/0004-637x/803/1/34.

28. Finkelstein, S.L.; Ryan, R.E.; Papovich, C.; Dickinson, M.; Song, M.; Somerville, R.S.; Ferguson, H.C.; Salmon, B.; Giavalisco, M.; Koekemoer, A.M.; Ashby, M.L.N.; Behroozi, P.; Castellano, M.; Dunlop, J.S.; Faber, S.M.; Fazio, G.G.; Fontana, A.; Grogin, N.A.; Hathi, N.; Jaacks, J.; Kocevski, D.D.; Livermore, R.; McLure, R.J.; Merlin, E.; Mobasher, B.; Newman, J.A.; Rafelski, M.; Tilvi, V.; Willner, S.P. The Evolution of the Galaxy Rest-Frame Ultraviolet Luminosity Function over the First Two Billion Years. The Astrophysical Journal 2015, 810, 71. doi:10.1088/0004-637x/810/1/71.

29. Atek, H.; Richard, J.; Jauzac, M.; Kneib, J.P.; Natarajan, P.; Limousin, M.; Schaerer, D.; Jullo, E.; Ebeling, H.; Egami, E.; Clement, B. Are Ultra-Faint Galaxies at $\mathrm{z}=6-8$ Responsible for Cosmic Reionization? Combined Constraints from the Hubble Frontier Fields Clusters and Parallels. The Astrophysical Journal 2015, 814, 69. doi:10.1088/0004-637x/814/1/69.

30. Livermore, R.C.; Finkelstein, S.L.; Lotz, J.M. Directly Observing the Galaxies Likely Responsible for Reionization. The Astrophysical Journal 2017, 835, 113. doi:10.3847/1538$4357 / 835 / 2 / 113$.

31. Finkelstein, S.L.; D'Aloisio, A.; Paardekooper, J.P.; Jr., R.R.; Behroozi, P.; Finlator, K.; Livermore, R.; Sanderbeck, P.R.U.; Vecchia, C.D.; Khochfar, S. Conditions for Reionizing the Universe with a Low Galaxy Ionizing Photon Escape Fraction. The Astrophysical Journal 2019, 879, 36. doi:10.3847/1538-4357/ab1ea8.

32. Yajima, H.; Choi, J.H.; Nagamine, K. Escape fraction of ionizing photons from high-redshift galaxies in cosmological SPH simulations. Monthly Notices of the Royal Astronomical Society 2011, 412, 411-422. doi:10.1111/j.1365-2966.2010.17920.x.

33. Lapi, A.; Danese, L. Cold or warm? Constraining dark matter with primeval galaxies and cosmic reionization after Planck. Journal of Cosmology and Astroparticle Physics 2015, 2015, 003-003. doi:10.1088/1475-7516/2015/09/003.

34. Haardt, F.; Madau, P. Radiative Transfer in a Clumpy Universe. IV. New Synthesis Models of the Cosmic UV/X-Ray Background. The Astrophysical Journal 2012, 746, 125 . doi: 10.1088/0004-637x/746/2/125. 
35. Pawlik, A.H.; Schaye, J.; Van Scherpenzeel, E. Keeping the Universe ionized: photoheating and the clumping factor of the high-redshift intergalactic medium. Monthly Notices of the Royal Astronomical Society 2009, 394, 1812-1824. doi:10.1111/j.1365-2966.2009.14486.x.

36. Bouwens, R.J.; Illingworth, G.D.; Oesch, P.A.; Caruana, J.; Holwerda, B.; Smit, R.; Wilkins, S. Reionization After Planck: the Derived Growth of the Cosmic Ionizing Emissivity now Matches the Growth of the Galaxy UV Luminosity Density. The Astrophysical Journal 2015, 811, 140. doi:10.1088/0004-637x/811/2/140.

37. King, A.; Pounds, K. Powerful Outflows and Feedback from Active Galactic Nuclei. Annual Review of Astronomy and Astrophysics 2015, 53, 115-154. doi:10.1146/annurev-astro-082214122316.

38. Menci, N.; Fiore, F.; Feruglio, C.; Lamastra, A.; Shankar, F.; Piconcelli, E.; Giallongo, E.; Grazian, A. Outflows in the Disks of Active Galaxies. The Astrophysical Journal 2019, 877, 74. doi:10.3847/1538-4357/ab1a3a.

39. Menci, N.; Fiore, F.; Puccetti, S.; Cavaliere, A. The Blast Wave Model for AGN Feedback: Effects on AGN Obscuration. The Astrophysical Journal 2008, 686, 219-229. doi:10.1086/591438.

40. Grissom, R. L..; Ballantyne, D. R..; Wise, J. H.. On the contribution of active galactic nuclei to reionization. AEA 2014, 561, A90. doi:10.1051/0004-6361/201322637.

41. Matsuoka, Y.; Strauss, M.A.; Kashikawa, N.; Onoue, M.; Iwasawa, K.; Tang, J.J.; Lee, C.H.; Imanishi, M.; Nagao, T.; Akiyama, M.; Asami, N.; Bosch, J.; Furusawa, H.; Goto, T.; Gunn, J.E.; Harikane, Y.; Ikeda, H.; Izumi, T.; Kawaguchi, T.; Kato, N.; Kikuta, S.; Kohno, K.; Komiyama, Y.; Lupton, R.H.; Minezaki, T.; Miyazaki, S.; Murayama, H.; Niida, M.; Nishizawa, A.J.; Noboriguchi, A.; Oguri, M.; Ono, Y.; Ouchi, M.; Price, P.A.; Sameshima, H.; Schulze, A.; Shirakata, H.; Silverman, J.D.; Sugiyama, N.; Tait, P.J.; Takada, M.; Takata, T.; Tanaka, M.; Toba, Y.; Utsumi, Y.; Wang, S.Y.; Yamashita, T. Subaru High-z Exploration of Lowluminosity Quasars (SHELLQs). V. Quasar Luminosity Function and Contribution to Cosmic Reionization at $\mathrm{z}=6$. The Astrophysical Journal 2018, 869, 150. doi:10.3847/1538-4357/aaee7a.

42. Lusso, E.; Worseck, G.; Hennawi, J.F.; Prochaska, J.X.; Vignali, C.; Stern, J.; O’Meara, J.M. The first ultraviolet quasar-stacked spectrum at $\mathrm{z} \simeq 2.4$ from WFC3. Monthly Notices of the Royal Astronomical Society 2015, 449, 4204-4220. doi:10.1093/mnras/stv516.

43. Calura, F.; Menci, N.; Gallazzi, A. The ages of stellar populations in a warm dark matter universe. Monthly Notices of the Royal Astronomical Society 2014, 440, 2066-2076. doi: 10.1093/mnras/stu339.

44. Grazian, A..; Giallongo, E..; Boutsia, K..; Cristiani, S..; Vanzella, E..; Scarlata, C..; Santini, P..; Pentericci, L..; Merlin, E..; Menci, N..; Fontanot, F..; Fontana, A..; Fiore, F..; Civano, F..; Castellano, M..; Brusa, M..; Bonchi, A..; Carini, R..; Cusano, F..; Faccini, M..; Garilli, B..; Marchetti, A..; Rossi, A..; Speziali, R.. The contribution of faint AGNs to the ionizing background at z 4. AEA 2018, 613, A44. doi:10.1051/0004-6361/201732385.

45. Mason, C.A.; Treu, T.; Dijkstra, M.; Mesinger, A.; Trenti, M.; Pentericci, L.; de Barros, S.; Vanzella, E. The Universe Is Reionizing at $\mathrm{z} \sim 7$ : Bayesian Inference of the IGM Neutral Fraction Using Ly $\alpha$ Emission from Galaxies. The Astrophysical Journal 2018, 856, 2. doi: 10.3847/1538-4357/aab0a7.

46. Konno, A.; Ouchi, M.; Ono, Y.; Shimasaku, K.; Shibuya, T.; Furusawa, H.; Nakajima, K.; Naito, Y.; Momose, R.; Yuma, S.; Iye, M. Accelerated Evolution of the Ly $\alpha$ Luminosity Function at $\mathrm{z} \gtrsim 7$ Revealed by the SUBARU Ultra-Deep Survey for Ly $\alpha$ Emitters at $\mathrm{z}=7.3$. The Astrophysical Journal 2014, 797, 16. doi:10.1088/0004-637x/797/1/16.

47. Konno, A.; Ouchi, M.; Shibuya, T.; Ono, Y.; Shimasaku, K.; Taniguchi, Y.; Nagao, T.; Kobayashi, M.A.R.; Kajisawa, M.; Kashikawa, N.; Inoue, A.K.; Oguri, M.; Furusawa, H.; Goto, T.; Harikane, Y.; Higuchi, R.; Komiyama, Y.; Kusakabe, H.; Miyazaki, S.; Nakajima, K.; Wang, S.Y. SILVERRUSH. IV. Ly $\alpha$ luminosity functions at $z=5.7$ and 6.6 studied with $\approx 1300 \mathrm{Ly} \alpha$ emitters on the $14-21 \mathrm{deg}^{2}$ sky. Publications of the Astronomical Society of Japan 2017, 70. S16, doi:10.1093/pasj/psx131.

48. Zheng, Z.Y.; Wang, J.; Rhoads, J.; Infante, L.; Malhotra, S.; Hu, W.; Walker, A.R.; Jiang, L.; Jiang, C.; Hibon, P.; Gonzalez, A.; Kong, X.; Zheng, X.; Galaz, G.; Barrientos, L.F. First Results from the Lyman Alpha Galaxies in the Epoch of Reionization (LAGER) Survey: Cosmological Reionization at $\mathrm{z} \sim 7$. The Astrophysical Journal 2017, 842, L22. doi:10.3847/2041-8213/aa794f.

49. Mesinger, A.; Aykutalp, A.; Vanzella, E.; Pentericci, L.; Ferrara, A.; Dijkstra, M. Can the intergalactic medium cause a rapid drop in Ly $\alpha$ emission at $\mathrm{z}>6$ ? Monthly Notices of the Royal Astronomical Society 2014, 446, 566-577. doi:10.1093/mnras/stu2089. 
50. Ouchi, M.; Harikane, Y.; Shibuya, T.; Shimasaku, K.; Taniguchi, Y.; Konno, A.; Kobayashi, M.; Kajisawa, M.; Nagao, T.; Ono, Y.; Inoue, A.K.; Umemura, M.; Mori, M.; Hasegawa, K.; Higuchi, R.; Komiyama, Y.; Matsuda, Y.; Nakajima, K.; Saito, T.; Wang, S.Y. Systematic Identification of LAEs for Visible Exploration and Reionization Research Using Subaru HSC (SILVERRUSH). I. Program strategy and clustering properties of $\approx 2000$ Ly $\alpha$ emitters at $z=6-7$ over the $0.3-0.5 \mathrm{Gpc}^{2}$ survey area. Publications of the Astronomical Society of Japan 2017, 70. S13, doi:10.1093/pasj/psx074.

51. Schenker, M.A.; Ellis, R.S.; Konidaris, N.P.; Stark, D.P. Line-Emitting Galaxies Beyond a Redshift of 7: an Improved Method for Estimating the Evolving Neutrality of the Intergalactic Medium. The Astrophysical Journal 2014, 795, 20. doi:10.1088/0004-637x/795/1/20.

52. Robertson, B.E.; Ellis, R.S.; Furlanetto, S.R.; Dunlop, J.S. Cosmic Reionization and Early Star-Forming Galaxies: a Joint Analysis of New Constraints from Planck and the Hubble Space Telescope. The Astrophysical Journal 2015, 802, L19. doi:10.1088/2041-8205/802/2/119.

53. Bouwens, R.J.; Smit, R.; Labbé, I.; Franx, M.; Caruana, J.; Oesch, P.; Stefanon, M.; Rasappu, N. The Lyman-Continuum Photon Production Efficiency $\xi_{\text {ion }}$ of $\mathrm{z} \sim 4-5$ Galaxies from IRACBased $\mathrm{H} \alpha$ Measurements: Implications for the Escape Fraction and Cosmic Reionization. The Astrophysical Journal 2016, 831, 176. doi:10.3847/0004-637x/831/2/176.

54. Yung, L.Y.A.; Somerville, R.S.; Popping, G.; Finkelstein, S.L. Semi-analytic forecasts for JWST - III. Intrinsic production efficiency of Lyman-continuum radiation. Monthly Notices of the Royal Astronomical Society 2020, 494, 1002-1017. doi:10.1093/mnras/staa714.

55. Dayal, P.; Volonteri, M.; Choudhury, T.R.; Schneider, R.; Trebitsch, M.; Gnedin, N.Y.; Atek, H.; Hirschmann, M.; Reines, A. Reionization with galaxies and active galactic nuclei. Monthly Notices of the Royal Astronomical Society 2020, 495, 3065-3078. doi:10.1093/mnras/staa1138.

56. Dayal, P.; Ferrara, A. Early galaxy formation and its large-scale effects. Physics Reports 2018, 780-782, 1-64. Early galaxy formation and its large-scale effects, doi: https://doi.org/10.1016/j.physrep.2018.10.002.

57. Euclid Collaboration. Euclid preparation - V. Predicted yield of redshift $7<\mathrm{z}<9$ quasars from the wide survey. AEA 2019, 631, A85. doi:10.1051/0004-6361/201936427. 
